\title{
Article
}

\section{JCMT BISTRO Survey Observations of the Ophiuchus Molecular Cloud: Dust Grain Alignment Properties Inferred Using a Ricean Noise Model}

Pattle, Kate, Lai, Shih-Ping, Hasegawa, Tetsuo, Wang, Jia-Wei, Furuya, Ray S., Ward-Thompson, Derek and Et, Al

Available at https://clok.uclan.ac.uk/29445/

Pattle, Kate, Lai, Shih-Ping, Hasegawa, Tetsuo, Wang, Jia-Wei, Furuya, Ray S., Ward-Thompson, Derek orcid iconORCID: 0000-0003-1140-2761 and Et, Al (2019) JCMT BISTRO Survey Observations of the Ophiuchus Molecular Cloud: Dust Grain Alignment Properties Inferred Using a Ricean Noise Model. The Astrophysical Journal, 880 (1). ISSN 0004-637X

It is advisable to refer to the publisher's version if you intend to cite from the work. http://dx.doi.org/10.3847/1538-4357/ab286f

For more information about UCLan's research in this area go to http://www.uclan.ac.uk/researchgroups/ and search for < name of research Group>.

For information about Research generally at UCLan please go to http://www.uclan.ac.uk/research/

All outputs in CLoK are protected by Intellectual Property Rights law, including Copyright law. Copyright, IPR and Moral Rights for the works on this site are retained by the individual authors and/or other copyright owners. Terms and conditions for use of this material are defined in the policies page. 


\title{
JCMT BISTRO Survey Observations of the Ophiuchus Molecular Cloud: Dust Grain Alignment Properties Inferred Using a Ricean Noise Model
}

\author{
Kate Pattle $^{1,2}$ (D), Shih-Ping Lai ${ }^{1,3}$ (D), Tetsuo Hasegawa ${ }^{2}$ (D) , Jia-Wei Wang ${ }^{1}$ (D), Ray S. Furuya ${ }^{4}$, Derek Ward-Thompson ${ }^{5}$ (D), \\ Pierre Bastien $^{6}$ (D), Simon Coudé ${ }^{7}$ (D), Chakali Eswaraiah ${ }^{8,9}$ (D), Lapo Fanciullo ${ }^{3}$ (D), James di Francesco ${ }^{10,11}$ (iD), Thiem Hoang ${ }^{12,13}$ (iD), \\ Gwanjeong Kim ${ }^{14}$ (D), Woojin Kwon ${ }^{12,13}$ (D), Chang Won Lee ${ }^{12,13}$ (D), Sheng-Yuan Liu ${ }^{3}$ (D), Tie Liu ${ }^{12,15}$ (D), \\ Masafumi Matsumura $^{16}$ (D), Takashi Onaka ${ }^{17,18}$ (D), Sarah Sadavoy ${ }^{19}$ (D), and Archana Soam ${ }^{7}$ (D) \\ ${ }^{1}$ Institute for Astronomy and Department of Physics, National Tsing Hua University, Hsinchu 30013, Taiwan; kpattle@ gapp.nthu.edu.tw \\ ${ }^{2}$ National Astronomical Observatory of Japan, National Institutes of Natural Sciences, Osawa, Mitaka, Tokyo 181-8588, Japan \\ ${ }^{3}$ Academia Sinica Institute of Astronomy and Astrophysics, P.O. Box 23-141, Taipei 10617, Taiwan \\ ${ }^{4}$ Institute of Liberal Arts and Sciences, Tokushima University, Minami Jousanajima-machi 1-1, Tokushima 770-8502, Japan \\ 5 Jeremiah Horrocks Institute, University of Central Lancashire, Preston PR1 2HE, UK \\ ${ }^{6}$ Centre de recherche en astrophysique du Québec \& département de physique, Université de Montréal, C.P. 6128 Succ. Centre-ville, Montréal, QC, H3C 3J7, Canada \\ ${ }_{8}^{7}$ SOFIA Science Center, Universities Space Research Association, NASA Ames Research Center, Moffett Field, CA 94035, USA \\ ${ }^{8}$ CAS Key Laboratory of FAST, National Astronomical Observatories, Chinese Academy of Sciences, People's Republic of China \\ ${ }^{9}$ National Astronomical Observatories, Chinese Academy of Sciences, Datun Road, Chaoyang District, Beijing 100101, People's Republic of China \\ ${ }^{10}$ NRC Herzberg Astronomy and Astrophysics, 5071 West Saanich Road, Victoria, BC V9E 2E7, Canada \\ ${ }^{11}$ Department of Physics and Astronomy, University of Victoria, Victoria, BC V8W 2Y2, Canada
${ }^{12}$ Korea Astronomy and Space Science Institute (KASI), 776 Daedeokdae-ro, Yuseong-gu, Daejeon 34055, Republic of Korea \\ ${ }^{14}$ Nobeyama Radio Observatory, National Astronomical Observatory of Japan, National Institutes of Natural Sciences, Nobeyama, Minamimaki, Minamisaku,
${ }^{13}$, \\ Nagano 384-1305, Japan \\ ${ }^{15}$ East Asian Observatory, 660 North A'ohōkū Place, University Park, Hilo, HI 96720, USA \\ ${ }^{16}$ Faculty of Education and Center for Educational Development and Support, Kagawa University, Saiwai-cho 1-1, Takamatsu, Kagawa, 760-8522, Japan \\ ${ }^{17}$ Department of Astronomy, Graduate School of Science, The University of Tokyo, 7-3-1 Hongo, Bunkyo-ku, Tokyo 113-0033, Japan \\ ${ }^{18}$ Faculty of Science and Engineering, Meisei University, 2-1-1 Hodokubo, Hino, Tokyo 191-856, Japan \\ ${ }^{19}$ Harvard-Smithsonian Center for Astrophysics, 60 Garden Street, Cambridge, MA 02138, USA \\ Received 2019 April 1; revised 2019 June 7; accepted 2019 June 7; published 2019 July 22
}

\begin{abstract}
The dependence of the polarization fraction $p$ on total intensity $I$ in polarized submillimeter emission measurements is typically parameterized as $p \propto I^{-\alpha}(\alpha \leqslant 1)$ and used to infer dust grain alignment efficiency in star-forming regions, with an index $\alpha=1$ indicating near-total lack of alignment of grains with the magnetic field. In this work, we demonstrate that the non-Gaussian noise characteristics of the polarization fraction may produce apparent measurements of $\alpha \sim 1$ even in data with significant signal-to-noise in Stokes $Q, U$, and $I$ emission, and so with robust measurements of polarization angle. We present a simple model demonstrating this behavior and propose a criterion by which well-characterized measurements of the polarization fraction may be identified. We demonstrate that where our model is applicable, $\alpha$ can be recovered by fitting the $p-I$ relationship with the mean of the Rice distribution without statistical debiasing of the polarization fraction. We apply our model to JCMT BISTRO Survey POL-2 $850 \mu \mathrm{m}$ observations of three clumps in the Ophiuchus molecular cloud, finding that in the externally illuminated Oph A region, $\alpha \approx 0.34$, while in the more isolated Oph B and C, despite their differing star formation histories, $\alpha \sim 0.6-0.7$. Our results thus suggest that dust grain alignment in dense gas is more strongly influenced by the incident interstellar radiation field than by star formation history. We further find that grains may remain aligned with the magnetic field at significantly higher gas densities than has previously been believed, thus allowing investigation of magnetic field properties within star-forming clumps and cores.
\end{abstract}

Key words: dust, extinction - ISM: magnetic fields - submillimeter: ISM - stars: formation - techniques: polarimetric

\section{Introduction}

The role of magnetic fields in the star formation process is not well understood (e.g., Crutcher 2012). Observations of magnetic field morphologies in the densest parts of molecular clouds are typically performed indirectly through submillimeter dust polarization observations (e.g., Matthews et al. 2009). In low-density, well-illuminated environments in the interstellar medium (ISM), dust grains are expected to be aligned with their minor axes parallel to the local magnetic field direction (Davis \& Greenstein 1951). However, at sufficiently high optical depths, grains are expected to become less efficiently aligned with the magnetic field (Andersson et al. 2015). Recent results have suggested that this occurs at a visual extinction
$A_{V} \sim$ 20-30 mag (Whittet et al. 2008; Alves et al. 2014; Jones et al. 2015). This inferred break in behavior is, in each case so far reported, coincident with a change in tracer from optical/ near-infrared extinction polarimetry to submillimeter dust emission polarimetry (Andersson et al. 2015; Jones et al. 2015). Submillimeter emission polarimetry is the only effective wide-area tracer of ISM polarization in dense molecular clouds $\left(A_{V} \gtrsim 30 \mathrm{mag}\right.$ ) where stars are forming. It is thus vital to studies of the role of magnetic fields in star formation to know which gas densities are being traced by dust polarization observations.

A commonly used method of assessing the alignment of grains is to determine the relationship between polarization efficiency and visual extinction (Whittet et al. 2008; Alves et al. 2014; 
Jones et al. 2015, 2016). In submillimeter studies, this is generally treated as a relationship between the polarization fraction $p$ and total submillimeter intensity $I$, as polarization efficiency is identical to the polarization fraction for optically thin emission (Alves et al. 2015), and the optically thin submillimeter total intensity is proportional to the visual extinction $A_{V}$ for a given temperature (see Jones et al. 2015; Santos et al. 2017). Recent comparisons between $p$ and submillimeter dust opacity measurements (an alternative proxy for $A_{V}$ ) suggest that the standard assumption in polarization studies of $I \propto A_{V}$ is likely to be too simplistic (Juvela et al. 2018). However, regardless of the exact nature of the relationship between $I$ and $A_{V}$, an accurate measurement of the $p-I$ relationship is required in order to interpret dust grain alignment properties.

It is expected that observations of dense material within molecular clouds will show a power-law dependence of $p$ on $I$, $p \propto I^{-\alpha}$ (Whittet et al. 2008). This index is expected to steepen as grains become increasingly poorly aligned with the magnetic field: an index of $\alpha=0$ would indicate equal grain alignment at all optical depths, an index of $\alpha=0.5$ is predicted for a cloud in which grain alignment decreases linearly with increasing optical depth, while an index of $\alpha=1$ is predicted for an environment in which all observed polarized emission is produced in a thin layer at the surface of the cloud, and grains at higher densities have no preferred alignment relative to the magnetic field (see Whittet et al. 2008; Jones et al. 2015).

Several recent studies of molecular clouds and starless cores have found power-law indices $\alpha$ in the range $0.5-1$, for example, $\alpha=0.7$ in OMC-3 and 0.8 in Barnard 1 (Matthews \& Wilson 2000), $0.64 \pm 0.01$ in CB 54 and $0.55 \pm 0.22$ in DC 253-1.6 (Henning et al. 2001), $0.83 \pm 0.01$ in NGC 2024 FIR 5 (Lai et al. 2002), $0.92 \pm 0.17$ in Pipe 109 (Alves et al. 2014, 2015), 0.84-1.02 in W51 (Koch et al. 2018), 1.0 in FeSt 1-457 (Kandori et al. 2018), 0.7 or 0.8 in Oph A (Kwon et al. 2018), 0.9 in Oph B (Soam et al. 2018), and 1.0 in Oph C (Liu et al. 2019). (Note that a larger value of $\alpha$ indicates a steeper negative slope.) In most of these cases, the data used have been selected to have a signal-to-noise ratio $(\mathrm{S} / \mathrm{N}) \geqslant 3$ in some combination of polarization fraction, polarized intensity, and total intensity. Recent improvements in instrumental sensitivity have allowed more stringent selection criteria: some recent observations have employed an $\mathrm{S} / \mathrm{N}$ cut of 20 in total intensity (Kwon et al. 2018; Soam et al. 2018). These results have generally been taken to suggest poor grain alignment within the densest parts of molecular clouds.

Polarized intensity and polarization fraction are both constrained to be positive quantities and so are characterized by Ricean statistics (Rice 1945; Serkowski 1958), albeit approximately so in the case of the polarization fraction, as discussed below. This results in a strong positive bias in the measured polarization fraction at low total intensities. Several methods of correcting for this bias have been proposed, of varying levels of sophistication (Wardle \& Kronberg 1974; Simmons \& Stewart 1985; Vaillancourt 2006; Quinn 2012; Montier et al. 2015a, 2015b; Vidal et al. 2016; Müller et al. 2017). These methods are collectively known as (statistical) debiasing. An alternative approach is to bypass the problem of characterizing the polarization fraction by working with the Stokes parameters of the observed polarized emission directly (e.g., Herron et al. 2018). The Rice distribution is discussed extensively in the electrical engineering literature due to its relevance to signal processing (e.g., Lindsey 1964; Sijbers et al. 1998; Abdi et al. 2001).
In this work, we investigate the extent to which measurements of the $p-I$ index are biased by the statistical behavior of the polarization fraction and choice of selection criteria. We demonstrate a method by which the relationship between $p$ and $I$ can be accurately characterized using the full observed data set without recourse to statistical debiasing.

The L1688 region of the Ophiuchus molecular cloud is a nearby (138.4 \pm 2.6 pc; Ortiz-León et al. 2018) site of low-tointermediate-mass star formation (Wilking et al. 2008). The region contains a number of dense clumps, Oph A-F, notable for their differing properties and star formation histories (Motte et al. 1998; Pattle et al. 2015). The Oph A, B, and C clumps have been observed with the James Clerk Maxwell Telescope (JCMT) POL-2 polarimeter as part of the JCMT B-fields in Star-forming Region Observations (BISTRO) survey (Ward-Thompson et al. 2017; Kwon et al. 2018; Soam et al. 2018; Liu et al. 2019). We apply the methods developed in this paper to the JCMT BISTRO survey observations of L1688.

This paper is structured as follows. In Section 2 we present the key equations governing the behavior of the polarization fractions. In Section 3, we present a simple model for the behavior of the polarization fraction as a function of $\mathrm{S} / \mathrm{N}$. In Section 4 we present Monte Carlo simulations demonstrating the behavior of our model and testing various fitting methods to recover the underlying relationship between polarization fraction and total intensity. In Section 5, we apply our model to recent JCMT POL-2 observations of the Ophiuchus molecular cloud. Section 6 summarizes our results.

\section{Mathematical Properties of the Polarization Fraction}

Linearly polarized intensity is given by

$$
P=\sqrt{Q^{2}+U^{2}}
$$

where $Q$ is the Stokes $Q$ intensity and $U$ is the Stokes $U$ intensity. We do not consider circular polarization here, and so we assume the Stokes $V$ intensity to be zero throughout. The polarization fraction, the ratio of polarized intensity $P$ to total intensity $I$, is given by

$$
p=\frac{\sqrt{Q^{2}+U^{2}}}{I} .
$$

We take measurements of Stokes $I, Q$, and $U$ to have measurement errors of $\delta I, \delta Q$, and $\delta U$, respectively. We assume that these measurement uncertainties are drawn from Gaussian distributions of width $\sigma_{I}, \sigma_{Q}$, and $\sigma_{U}$.

Note that all symbols defined and used in this work are summarized in Table 3 in the Appendix.

\subsection{The Rice Distribution}

Polarized intensity - the result of the addition in quadrature of real numbers, as shown in Equation (1) - must be a positive quantity. The addition in quadrature of small values of $Q$ and $U$, with measurement uncertainties $\delta Q$ and $\delta U$ will produce spurious measurements of polarized intensity where $\delta Q \gtrsim|Q|$ and $\delta U \gtrsim|U|$ produce spurious measurements of polarized intensity. The results of such an addition in quadrature are described mathematically by the Rice distribution (Rice 1945), where the quantities under addition have noise properties that are Gaussian and uncorrelated. In principle, we can expect the uncertainties on Stokes $Q$ and $U$ to be independent, as $Q$ and $U$ 
are orthogonal components of the Stokes polarization vector (see, e.g., Herron et al. 2018). We discuss the validity of this assumption in the specific case of JCMT POL-2 observations in Section 5 .

The Rice distribution has positive skewness at low $\mathrm{S} / \mathrm{N}$, while at high $\mathrm{S} / \mathrm{N}$, it tends toward a Gaussian distribution (Rice 1945). Assuming that the Stokes $Q$ and $U$ data have independent Gaussian measurement uncertainties, polarized intensities derived using Equation (1) will be Rice-distributed (Wardle \& Kronberg 1974; Simmons \& Stewart 1985).

As the distributions of the observed values of the polarized intensity, and so of the polarization fraction, are different from the underlying distributions that would be seen in the absence of measurement error, we henceforth denote the observed values of the polarized intensity and polarization fraction as $P^{\prime}$ and $p^{\prime}$, respectively.

The division $p^{\prime}=P^{\prime} / I$ (see Equation (2)) can, at low $\mathrm{S} / \mathrm{N}$ (where $I$ is small and $P^{\prime}$ may be artificially large), produce artificially high values of $p^{\prime}$. If $P^{\prime} / \delta P \ll I / \delta I$, as is generally the case, as $p \ll 1$ in physically realistic situations, the effect of the measurement error on $I$ on the distribution of $p^{\prime}$ will be minimal, so $p^{\prime}$ will also be approximately Rice-distributed (see Simmons \& Stewart 1985).

If the polarization fractions that we measure can be treated as being Rice-distributed, the probability of measuring a polarization fraction $p^{\prime}$ given a true polarization fraction $p$ and an rms uncertainty in polarization fraction $\sigma_{p}$ (the probability density function) is

$$
\operatorname{PDF}_{\text {Rice }}\left(p^{\prime} \mid p\right)=\frac{p^{\prime}}{\sigma_{p}^{2}} \exp \left(-\frac{p^{\prime 2}+p^{2}}{2 \sigma_{p}^{2}}\right) \mathcal{I}_{0}\left(\frac{p^{\prime} p}{\sigma_{p}^{2}}\right),
$$

where $\mathcal{I}_{0}$ is the zeroth-order modified Bessel function (Simmons \& Stewart 1985). See Montier et al. (2015a) for a derivation of this result (see also Bastien et al. 2007; Hull \& Plambeck 2015).

The mean of the Rice distribution is given by

$$
\mu_{p}=\sqrt{\frac{\pi}{2}} \sigma_{p} \mathcal{L}_{\frac{1}{2}}\left(-\frac{p^{2}}{2 \sigma_{p}^{2}}\right),
$$

where $\mathcal{L}_{\frac{1}{2}}$ is a Laguerre polynomial of order $\frac{1}{2}$,

$$
\begin{aligned}
\mathcal{L}_{\frac{1}{2}}\left(-\frac{p^{2}}{2 \sigma_{p}^{2}}\right)= & e^{-\frac{p^{2}}{4 \sigma_{p}^{2}}}\left[\left(1+\frac{p^{2}}{2 \sigma_{p}^{2}}\right) \mathcal{I}_{0}\left(\frac{p^{2}}{4 \sigma_{p}^{2}}\right)\right. \\
& \left.+\frac{p^{2}}{2 \sigma_{p}^{2}} \mathcal{I}_{1}\left(\frac{p^{2}}{4 \sigma_{p}^{2}}\right)\right]
\end{aligned}
$$

and $\mathcal{I}_{0}$ and $\mathcal{I}_{1}$ are modified Bessel functions of order zero and 1 , respectively.

We note that approximating $p^{\prime}$ as Rice-distributed is a statement that $I$ is perfectly known, and so is equivalent to setting its rms uncertainty $\sigma_{I}=0$. We will demonstrate in Section 4 that Equation (3) describes synthetic data with realistic noise properties sufficiently well to justify making this assumption.

Having chosen to treat $I$ as perfectly known, if the measurement uncertainties on $Q$ and $U$ are equivalent, i.e., $\sigma_{Q}=\sigma_{U}$, then $\sigma_{p}$ will be given by

$$
\sigma_{p}=\frac{\sigma_{Q}}{I}=\frac{\sigma_{U}}{I}
$$

(see Equation (2)). This approximation is discussed by Montier et al. (2015a).

\subsection{Debiasing}

The correction of the observed polarization fraction $p^{\prime}$ for the bias described above is known as "debiasing." A commonly used method for debiasing polarization fractions is the Wardle \& Kronberg (1974) estimator (see also Serkowski 1962), under which the debiased observed polarized intensity is given by

$$
P_{\mathrm{db}}^{\prime}=\sqrt{Q^{2}+U^{2}-\frac{1}{2}\left(\delta Q^{2}+\delta U^{2}\right)} .
$$

Similarly, the debiased observed polarization fraction is given by

$$
p_{\mathrm{db}}^{\prime}=\frac{\sqrt{Q^{2}+U^{2}-\frac{1}{2}\left(\delta Q^{2}+\delta U^{2}\right)}}{I} .
$$

As this is the default debiasing method for POL-2 data (e.g., Kwon et al. 2018), we compare the results of fitting nondebiased data and data debiased using the Wardle \& Kronberg (1974) estimator in Section 4. For a detailed comparison of debiasing methods, see Montier et al. (2015b).

\subsection{An Aside on Polarization Angle}

The polarization angle $\theta_{p}$ is given by

$$
\theta_{p}=\frac{1}{2} \arctan (U, Q) \text {. }
$$

We note that measurements of the polarization angle follow a different probability distribution than those of the polarization fraction (Naghizadeh-Khouei \& Clarke 1993). We expect measurements of the polarization angle to be significantly more robust than those of the polarization fraction, as the $Q$ and $U$ distributions have the same statistical properties and thus their ratio is not affected by the issues discussed above. Note also that the polarization angle is not constrained to be positive.

Inference of grain alignment properties from the polarization angle distribution would require at minimum a model of the plane-of-sky magnetic field morphology and an estimate of the fluctuations in magnetic field direction induced by Alfvénic turbulence (Chandrasekhar \& Fermi 1953). We therefore do not consider polarization angles in this work.

\section{A Simple Model for the Polarization Fraction}

We construct a simple model in which the polarization fraction is fully described by

$$
p(I)=p_{0}\left(\frac{I}{I_{0}}\right)^{-\alpha}
$$

where $p_{0}$ is the polarization fraction at the reference intensity, $I_{0}$. We expect $0<\alpha \leqslant 1$ (e.g., Whittet et al. 2008).

The reference intensity, or normalization, $I_{0}$, can be treated as a free parameter of the model. However, we choose to specify $I_{0}=\sigma_{Q U}$, where $\sigma_{Q U}$ is a single value, with units of intensity, representative of the rms noise in both Stokes $Q$ and $U$ measurements. We thus assume that the Stokes $Q$ and $U$ data sets have identical statistical properties and can be adequately characterized by a single rms noise value. This is a reasonable assumption for recent submillimeter emission polarization measurements, as described in Section 5 below. 
By taking $I_{0}=\sigma_{Q U}$, we assume that the power-law relationship between $I$ and $p$ applies to all measurements above the noise level of the data. Choosing $I_{0}=\sigma_{Q U}$ allows us to discuss the behavior of our model in terms of a simple $\mathrm{S} / \mathrm{N}$ criterion, $I / \sigma_{Q U}$, as demonstrated below. The reference polarization fraction $p_{0}$ thus depends on $\sigma_{Q U}$. In order to emphasize this, we define the polarization fraction at the noise level of the data, $p\left(I=\sigma_{Q U}\right)=$ $p_{\sigma_{Q U}}$; i.e., if $I_{0}=\sigma_{Q U}, p_{0}=p_{\sigma_{Q U}}$.

In order to meaningfully compare polarization fractions in data sets with different rms noise levels, we must convert $p_{\sigma_{Q U}}$ to the polarization fraction at a common reference intensity level. In this work, we choose a reference intensity of $I=100 \mathrm{mJy}^{\text {beam }}{ }^{-1}$ and so define

$$
p_{100 \mathrm{mJy}_{\text {beam }}^{-1}}=p_{\sigma_{Q U}}\left(\frac{100 \mathrm{mJy} \mathrm{beam}^{-1}}{\sigma_{Q U}}\right)^{-\alpha} .
$$

The choice to reference to $100 \mathrm{mJy}^{\text {beam }}{ }^{-1}$ is largely arbitrary but suitable for the JCMT POL-2 data that we consider in Section 5 below, in which $I=100 \mathrm{mJy} \mathrm{beam}^{-1}$ is both significantly above the rms noise level of the data and below the maximum intensities observed. Note that the JCMT has an effective beam size of $14 ! " 1$ at $850 \mu \mathrm{m}$ (Dempsey et al. 2013).

Equation (10) becomes unphysical where $p(I)>1$. Moreover, in physically realistic scenarios, we expect $p \lesssim 0.2$ in data with good $\mathrm{S} / \mathrm{N}$, as the maximum percentage of polarization observed in the diffuse ISM is $\sim 20 \%$ (Planck Collaboration et al. 2015). We thus require that $p<1$ and expect that $p \lesssim 0.2$ wherever we believe our observed Stokes $Q, U$, and $I$ values to be reliable.

\subsection{Physical Implications of $\mathrm{a}$}

If $\alpha=0$, this implies that the polarized intensity $P=$ $\sqrt{Q^{2}+U^{2}} \propto I$, so $p=p_{\sigma_{Q U}}$ everywhere. If polarized intensity is directly proportional to total intensity, this indicates that all emission along each sight line is polarized to the same degree, so there is no variation in polarization efficiency anywhere within the observed cloud.

If $\alpha=1$, this implies that $P$ does not vary with $I$; i.e., a constant amount of polarized emission is observed at all locations in the cloud. This indicates that only a small portion of the total line of sight is contributing polarized emission. This is usually interpreted as a thin layer of polarized emission overlaying an otherwise unpolarized optically thin sight line. This polarized emission is implicitly from low-density material; so, if the higher-density material along the sight line is also polarized, that polarized emission ought to also be detectable. Thus, claiming a physical index of $\alpha=1$ is essentially a statement that one has observed statistical noise in Stokes $Q$ and $U$ around a constant level of polarized intensity, and that this indicates a genuine absence of polarized emission at high total intensities, rather than insufficient $\mathrm{S} / \mathrm{N}$ to achieve a detection.

Intermediate values of $0<\alpha<1$ indicate a positive relationship, $P \propto I^{1-\alpha}$, in which the total amount of polarized emission increases with, but slower than, total emission. This implies that as the amount of material along the sight line increases, the ability of that material to produce polarized emission decreases (under the assumption of isothermal, optically thin emission). Thus, an index of $0<\alpha<1$ indicates that depolarization increases with depth into the cloud.

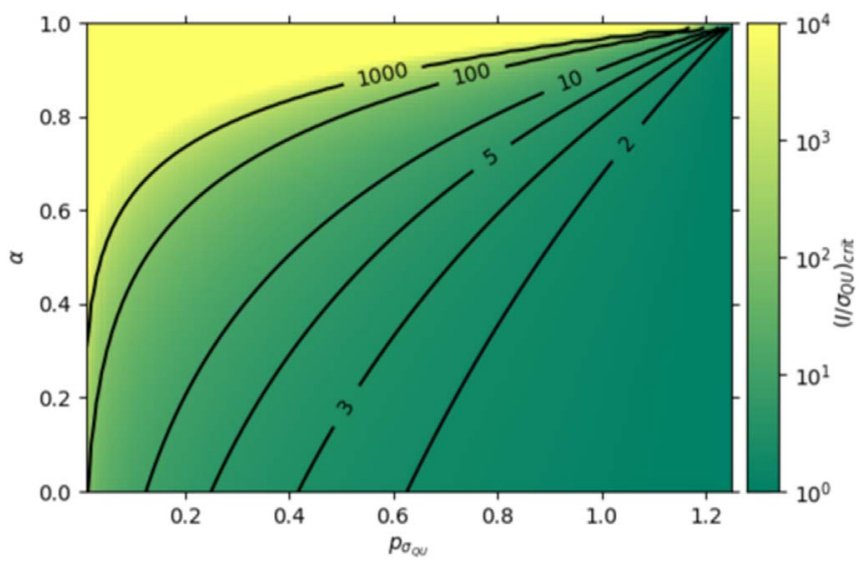

Figure 1. The $\mathrm{S} / \mathrm{N}$ value $\left(I / \sigma_{Q U}\right)_{\text {crit }}$ at which our model predicts a break in the power-law index as a function of $\alpha$ and $p_{\sigma_{Q U}}$, derived using Equation (14). In order to detect and accurately characterize a power-law behavior shallower than $\alpha=1$, a reasonable number of data points with $\left(I / \sigma_{Q U}\right)>\left(I / \sigma_{Q U}\right)_{\text {crit }}$ are required. The contours show lines of constant $\mathrm{S} / \mathrm{N}$. The color table saturates at $I / \sigma_{Q U}=10^{4}$. Note that not all combinations of $\alpha$ and $p_{\sigma_{Q U}}$ will produce physically plausible results.

\subsection{Low-S/N Limit}

In the low-S/N limit, the distribution of $p^{\prime}$ tends to the Rice distribution regardless of the value of $\alpha$ (or $p_{0}$ or $I_{0}$ ). In the limit where $p \ll \sigma_{p}, \mathcal{L}_{\frac{1}{2}}\left(-p^{2} / 2 \sigma_{p}\right) \rightarrow 1$, and therefore $\mu_{p} \rightarrow \sigma_{p} \sqrt{\pi / 2}$. Taking $\sigma_{p}=\sigma_{Q U} / I$, the behavior of the distribution is well approximated by

$$
p^{\prime}=\sqrt{\frac{\pi}{2}}\left(\frac{I}{\sigma_{Q U}}\right)^{-1}
$$

at small $I$. Thus, at low $\mathrm{S} / \mathrm{N}, p^{\prime} \propto I^{-1}$, regardless of the true value of $\alpha$.

In the no-signal case ( $p_{\sigma_{Q U}}=0 ; Q=U=0$ ), Equation (12) characterizes the observed signal at all values of $I$. Thus, in the absence of a true measurement, $p^{\prime} \propto I^{-1}$ behavior will be observed.

\subsection{High-S/N Limit}

In the high-S/N limit, the distribution of $p^{\prime}$ tends toward a Gaussian distribution around the true value,

$$
p^{\prime}=p_{\sigma_{Q U}}\left(\frac{I}{\sigma_{Q U}}\right)^{-\alpha} \text {. }
$$

\subsection{S/N Criterion}

Equations (12) and (13) suggest that, for $\alpha<1$, the underlying power-law dependence of $p$ on $I$ will be observable when

$$
\frac{I}{\sigma_{Q U}}>\left(\frac{I}{\sigma_{Q U}}\right)_{\text {crit }}=\left(\frac{1}{p_{\sigma_{Q U}}} \sqrt{\frac{\pi}{2}}\right)^{\frac{1}{1-\alpha}} .
$$

At $\mathrm{S} / \mathrm{Ns}$ below this critical value, the artificial $p^{\prime} \propto I^{-1}$ behavior will dominate, and the true value of $\alpha$ will not be recoverable.

Figure 1 shows solutions of Equation (14) for the critical $\mathrm{S} / \mathrm{N}$ value $\left(I / \sigma_{Q U}\right)_{\text {crit }}$, above which the true power-law behavior 
(i.e., the true value of $\alpha$ ) would be recoverable. It is apparent that significant $\mathrm{S} / \mathrm{N}$ is required to distinguish a power-law behavior with a steep value of $\alpha$ from instrumental noise.

\section{Monte Carlo Simulations}

In order to test the accuracy of our interpretation of the model described above, we performed a set of Monte Carlo simulations.

We investigated values of $\alpha$ in the range $0 \leqslant \alpha \leqslant 1$. In each case, we took $p_{100 \mathrm{mJy}_{\text {beam }}^{-1}}=0.025$ (i.e., emission is intrinsically $2.5 \%$ polarized at $I=100.0$; intensity units are arbitrary but chosen to mimic POL-2 data, which are calibrated in mJy beam $\left.^{-1}\right)$. This value of $p_{100 ~} \mathrm{mJy}_{\text {beam-1 }}$ was chosen for its similarity to the POL-2 observations. We then calculated the appropriate value of $p_{\sigma_{Q U}}$ for our chosen $\alpha$ and $p_{100 \mathrm{mJy}_{\text {beam }}{ }^{-1} \text { using }}$ Equation (11).

As we are not concerned with polarization angles in this work, we choose for simplicity that Stokes $U=0\left(\theta_{p}=0^{\circ}\right.$, implying that the underlying magnetic field is uniform and oriented $90^{\circ}$ east of north), and so Equation (10) is equivalent to

$$
Q(I)=p(I) \times I=p_{\sigma_{Q U}} \sigma_{Q U}^{\alpha} I^{1-\alpha}
$$

We drew a set of $10^{3}$ randomly distributed $\log _{10}\left(I / \sigma_{Q U}\right)$ values in the range $0<\log _{10}\left(I / \sigma_{Q U}\right)<3$ (thus assuming that low-S/N values of $I$ are more probable). We drew measurement errors $\Delta Q$ and $\Delta U$ on Stokes $Q$ and $U$ from Gaussian distributions of equal (fixed) width, $\sigma_{Q U}$, and $\Delta I$ on Stokes $I$ from a Gaussian distribution of (fixed) width $\sigma_{I}$. We here chose $\sigma_{Q U}=\sigma_{I}=5.0$ (arbitrary units). Equations (3) and (4) assume that the effect of $\sigma_{I}$ on the distribution of $p$ is negligible. We wished to test whether that approximation is valid in observations where the noise on Stokes $I$ is comparable to that on Stokes $Q$ and $U$.

The "observed" values $Q_{\text {obs }}, U_{\text {obs }}$, and $I_{\text {obs }}$ were then, for the ith value,

$$
\begin{gathered}
Q_{\mathrm{obs}, i}=Q_{i}+\Delta Q_{i}, \\
U_{\mathrm{obs}, i}=\Delta U_{i},
\end{gathered}
$$

and

$$
I_{\mathrm{obs}, i}=I_{i}+\Delta I_{i}
$$

"Observed" values of $p^{\prime}$ and $p_{\mathrm{db}}^{\prime}$ were calculated using Equations (2) and (8), respectively, for a given value of $p_{\sigma_{Q U}}$. We repeated this process $10^{4}$ times.

Figure 2 shows a single realization of our Monte Carlo simulations for three cases: $\alpha=1,0.5$, and 0 (constant polarization fraction). In all cases, $p_{100 \mathrm{mJy}_{\text {beam }}{ }^{-1}}=0.025$. The results are shown without any debiasing of the polarization fraction and colored according to their $\mathrm{S} / \mathrm{N}$ in $I$ and $p^{\prime}$.

Figure 2 shows that where $I / \sigma_{Q U}<\left(I / \sigma_{Q U}\right)_{\text {crit }}$, the distribution shows an identical $p^{\prime} \propto I^{-1}$ behavior in all cases, and that the underlying power-law behavior does not dominate over the $I^{-1}$ dependence until $I / \sigma_{Q U} \gg\left(I / \sigma_{Q U}\right)_{\text {crit. }}$. Thus, unless a reasonable number of data points have $\mathrm{S} / \mathrm{Ns}$ significantly greater than the critical value, the true powerlaw behavior is not recoverable. The overall behavior of the recovered polarization fraction is well described by the mean of the Rice distribution.
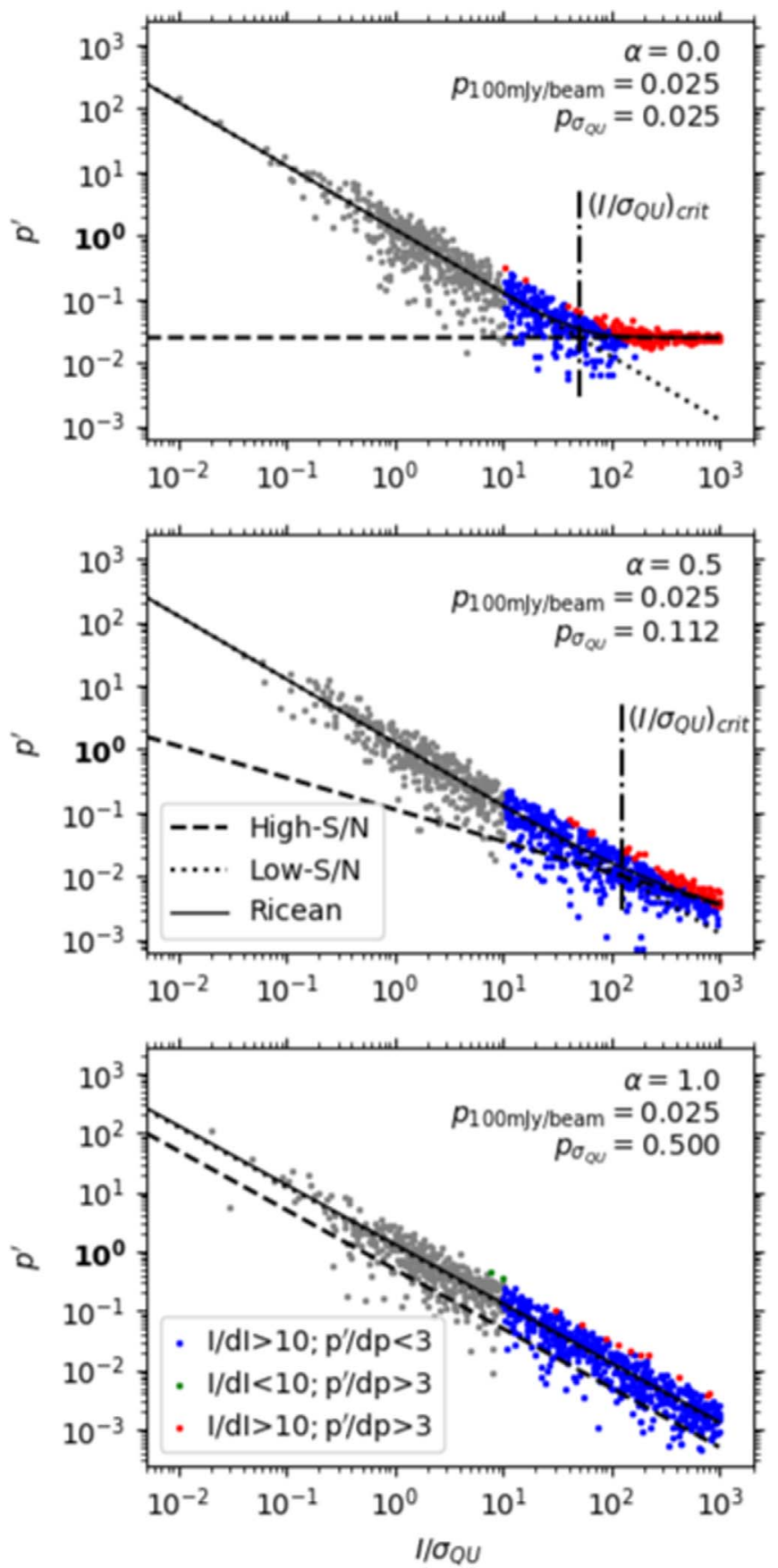

Figure 2. Monte Carlo simulations of the observed polarization fraction $p^{\prime}$ as a function of $\mathrm{S} / \mathrm{N} I / \sigma_{Q U}$ for three cases: $\alpha=0$ (top), 0.5 (middle), and 1 (bottom). In all cases, $p_{100 \mathrm{mJy}_{\text {beam }}{ }^{-1}}=0.025$. Results are shown without debiasing. The polarization fraction $p^{\prime}$ is given as an absolute value (not as a percentage), so any value $p^{\prime}>1$ (marked in bold on plot axes) is unphysical. Points are colored according to their $\mathrm{S} / \mathrm{N}$ in $I$ and $p^{\prime}$; gray points meet none of the criteria shown in the legend. The solid black line shows $p^{\prime}=\mu_{p}$, the mean of the Rice distribution, for the given values of $\alpha$ and $p_{\sigma_{Q U}}$. The dashed black line shows the true polarization fraction $p=p_{\sigma_{Q U}}\left(I / \sigma_{Q U}\right)^{-\alpha}$. The dotted line shows the nullhypothesis/low-S/N relation $p^{\prime}=\sqrt{\pi / 2}\left(I / \sigma_{Q U}\right)^{-1}$. In the cases where $\alpha<1$, the dotted-dashed line marks the critical value $\left(I / \sigma_{Q U}\right)_{\text {crit }}=\left(p_{\sigma_{Q U}}^{-1} \sqrt{\pi / 2}\right) \frac{1}{1-\alpha}$.

\subsection{Uncertainty on Polarization Fraction}

In keeping with standard practice in observational polarimetry and the default behavior of the POL-2 pipeline (e.g., Kwon et al. 2018), we estimated the uncertainty on the 
polarization fraction using the relation

$$
\delta p=\left(\frac{Q^{2} \delta Q^{2}+U^{2} \delta U^{2}}{I^{2}\left(Q^{2}+U^{2}\right)}+\frac{\delta I^{2}\left(Q^{2}+U^{2}\right)}{I^{4}}\right)^{\frac{1}{2}}
$$

(e.g., Wardle \& Kronberg 1974). We note that this result is derived using classical error propagation and so assumes that $\delta Q, \delta U$, and $\delta I$ are small and uncorrelated. We here take $\delta Q=\delta U=\sigma_{Q U}$ and $\delta I=\sigma_{I}$. As discussed above, measurement errors $\delta p$ calculated using Equation (19) can only be treated as representative of a Gaussian distribution around $p^{\prime}$ when $p^{\prime} / \delta p$ is large.

\subsection{Fitting Methods}

We investigated the results of fitting two models to our Monte Carlo simulations. Fitting was performed using the scipy routine curve_fit. In all cases, we attempted to recover the input values of $p_{\sigma_{Q U}}$ and $\alpha$, assuming that $\sigma_{Q U}$ is a fixed and directly measurable property of the data set. We supplied our calculated $\delta p$ values to curve fit as $1 \sigma$ uncertainties on $p^{\prime}$. Although this is technically valid only at high $\mathrm{S} / \mathrm{N}$, the default behavior of this fitting routine is to consider only the relative magnitudes of the input uncertainties. The uncertainties provided thus down-weight the contribution of low-S/N points to the fitting process.

Single power-law-In keeping with standard practice, we fitted a single power-law model, Equation (13), to the high-S/ $\mathrm{N}$ data. This model assumes that $p^{\prime} \approx p$ and so is usually applied to data that have been statistically debiased. Applying this model to data that have not been debiased would inherently lead to artificially high values of $\alpha$ being recovered. In order to fairly test this model, we thus applied it to the debiased polarization fractions $p_{\mathrm{db}}^{\prime}$ returned by our Monte Carlo simulations; i.e., we fitted

$$
p_{\mathrm{db}}^{\prime}=p_{\sigma_{Q U}}\left(\frac{I}{\sigma_{Q U}}\right)^{-\alpha}
$$

to a high-S $/ \mathrm{N}$ subset of the debiased data. We tested two commonly used S/N criteria: $I / \delta I>10$ and $p_{\mathrm{db}}^{\prime} / \delta p>3$. As we are here selecting higher-S/N data points, the $\delta p$ values we use should be somewhat representative of $1 \sigma$ Gaussian uncertainties on $p_{\mathrm{db}}^{\prime}$. However, this model will provide accurate values of $p_{\sigma_{Q U}}$ and $\alpha$ only if the $p_{\mathrm{db}}^{\prime}$ values selected are well within the high-S/N limit described in Section 3.3.

Mean of Rice distribution-We fitted the mean of the Rice distribution (Equation (4)), assuming that $p$ is given by Equation (13), and that $\sigma_{p} \approx \sigma_{Q U} / I$, i.e.,

$$
p^{\prime}=\sqrt{\frac{\pi}{2}}\left(\frac{I}{\sigma_{Q U}}\right)^{-1} \mathcal{L}_{\frac{1}{2}}\left[-\frac{p_{\sigma_{Q U}}^{2}}{2}\left(\frac{I}{\sigma_{Q U}}\right)^{2(1-\alpha)}\right] .
$$

We hereafter refer to this model as the "Ricean-mean model." This model is applied to the entire data set without any selection by $\mathrm{S} / \mathrm{N}$ and to data that have not been statistically debiased. The Ricean-mean model is predicated on the Ricean probability density function (Equation (3)) being applicable to the data, which will not be the case if any attempt has been made to correct the data for observational bias. We note that although the $\delta p$ values used in the fitting process do not strictly represent $1 \sigma$ Gaussian uncertainties, they do have the effect of significantly down-weighting the contribution of low-S/N data to the best-fit model. Fitting this model thus requires a good measurement of $\sigma_{Q U}$ with which to constrain the low-S/N behavior.

We fitted these models to non-debiased (Ricean-mean) and debiased (power-law) values of $p^{\prime}$ for each realization of our Monte Carlo simulations. We tested values of $\alpha$ in the range $0 \leqslant \alpha \leqslant 1$. Our results are shown in Figure 3. We find that, of the fitting models we tested, only the Ricean-mean model can accurately recover both $\alpha$ and $p_{\sigma_{Q U}}$ when $\alpha$ is large.

For data with a maximum $I / \sigma_{Q U}$ value of 1000 and $p_{100 \mathrm{mJy}_{\text {beam }}^{-1}}=0.025, \alpha$ and $p_{\sigma_{Q U}}$ can be approximately recovered with the single power-law model only while $\alpha<0.3$. For the single power-law model, the recovered value of $\alpha$ is systematically larger than the input value, tending toward 1 as $\alpha$ increases (thus, an input value $\alpha=1$ can be accurately recovered). The recovered value of $p_{\sigma_{Q U}}$ is also larger than the input value for steep values of $\alpha$. Selecting $p / \delta p>3$ systematically increases the recovered value of $p_{\sigma_{Q U}}$, as would be expected from examination of Figure 2. Thus, we expect fitting a single power-law model to, in general, systematically return larger-than-input values of $\alpha$ and $p_{\sigma_{Q U}}$ and so overestimate the extent to which depolarization is occurring. This is shown in Figure 3.

We find that the Ricean-mean model performs well for most values of $\alpha$. As expected, the Ricean-mean model cannot accurately recover the input model parameters without a significant number of data points above $\left(I / \sigma_{Q U}\right)_{\text {crit }}$. If the data set under consideration has a maximum $\mathrm{S} / \mathrm{N}\left(I / \sigma_{Q U}\right)_{\max }$, a necessary condition for the input model parameters to be recoverable will be

$$
\left(\frac{I}{\sigma_{Q U}}\right)_{\max }>\left(\frac{I}{\sigma_{Q U}}\right)_{\text {crit }},
$$

because if the break from $p^{\prime} \propto I^{-1}$ to $p^{\prime} \propto I^{-\alpha}$ occurs above the maximum $\mathrm{S} / \mathrm{N}$ in the data set, $\alpha$ will perforce not be recoverable. Thus, for a given value of $p_{\sigma_{Q U}}$, there will be some theoretical maximum (i.e., steepest) recoverable value of $\alpha$, which we define as $\alpha_{\max }$, associated with $\left(I / \sigma_{Q U}\right)_{\max }=\left(I / \sigma_{Q U}\right)_{\text {crit }}$. Combining this equality with Equation (14) (substituting $\alpha_{\max }$ for $\alpha$ in the latter), we find

$$
\alpha_{\max }=1-\frac{\log \left(\frac{1}{p_{\sigma_{Q U}}} \sqrt{\frac{\pi}{2}}\right)}{\log \left(\left[\frac{I}{\sigma_{Q U}}\right]_{\max }\right)} .
$$

In practice, a reasonable number of data points must have $\mathrm{S} / \mathrm{Ns}$ greater than $\left(I / \sigma_{Q U}\right)_{\text {crit }}$ in order to accurately recover the input model values. Figure 3 shows that, for our chosen values of $p_{100 \mathrm{mJy}_{\text {beam }}-1}=0.025, \sigma_{Q U}=5.0$, and $\left(I / \sigma_{Q U}\right)_{\max }=1000$, the Ricean-mean model can accurately recover values up to $\alpha \approx 0.6$ and approximately recover $\alpha \approx 0.7$, but values steeper than this cannot be recovered accurately. The $\alpha=0.9$ and 1.0 cases have no data points above $\left(I / \sigma_{Q U}\right)_{\text {crit. }}$ Our results suggest that in order to accurately recover the input parameters, $\gtrsim 40 \%$ of the data points must be above $\left(I / \sigma_{Q U}\right)_{\text {crit }}$.

When performing these Monte Carlo simulations, we set $\sigma_{I}=\sigma_{Q U}$. We find that the Ricean-mean model can accurately recover the input model values despite the approximation $\sigma_{I}=0$ in Equation (3). This supports our assumption that the 

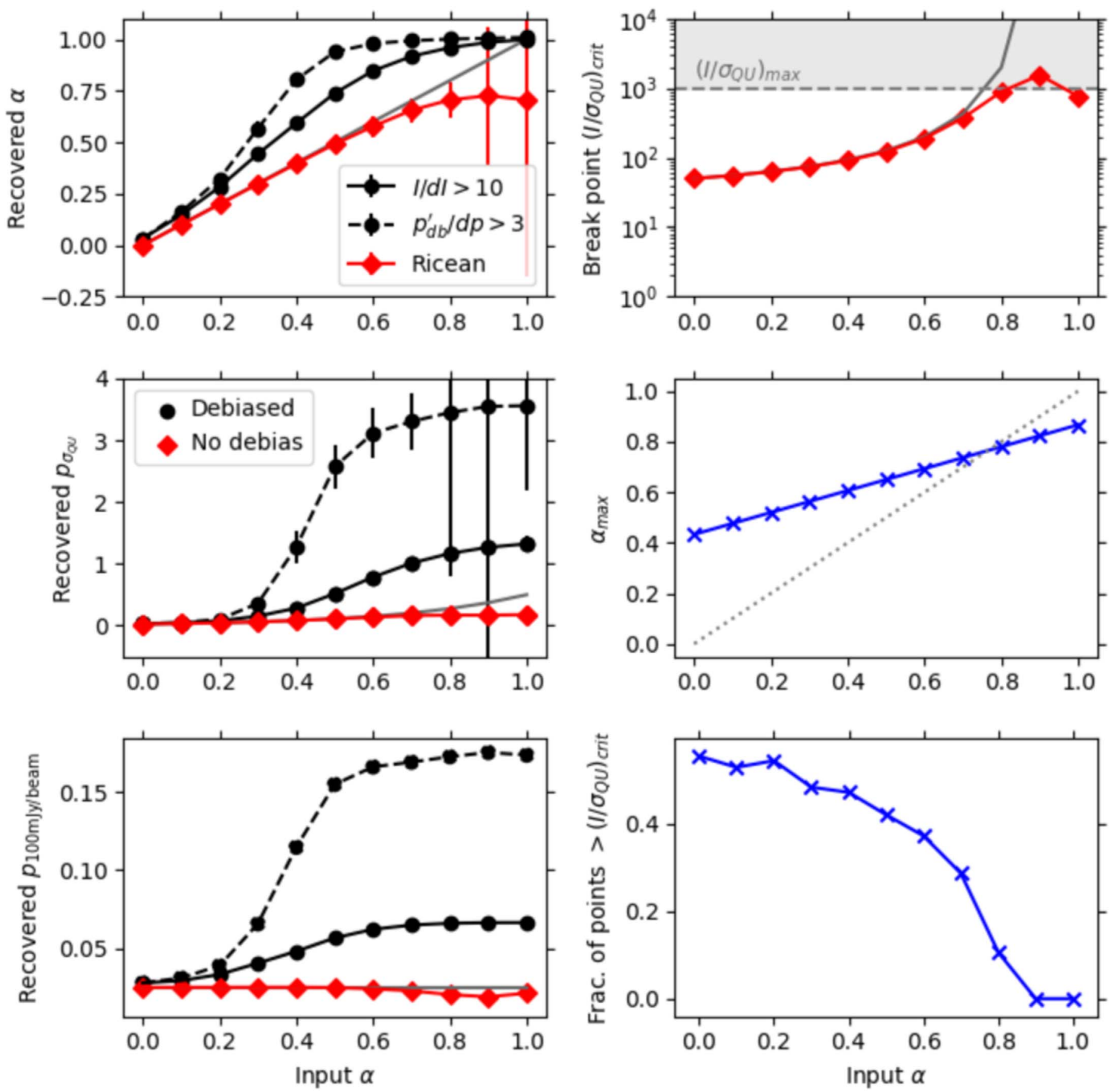

Figure 3. Results of fitting to Monte Carlo simulations for $p_{100 ~ \mathrm{mJy}_{\text {beam }}{ }^{-1}}=0.025$ and $0 \leqslant \alpha \leqslant 1$. Left column: The black data points show single power-law fitting to debiased data where $p / \delta p>3$ (dashed line) and $I / \delta I>10$ (solid line). The red data points show fitting of the Ricean-mean model to non-debiased data. Top row:

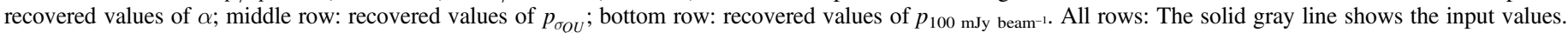
Right column (relevant to Ricean-mean model only): The top row shows the critical S/N value $\left(I / \sigma_{Q U}\right)_{\text {crit }}$ inferred from the best-fit $\alpha$ and $p_{\sigma Q U}$ for the Ricean-mean model. The solid gray line shows the input values. The horizontal dashed line shows the maximum $\mathrm{S} / \mathrm{N}$ value in the simulations, $\left(I / \sigma_{O U}\right)_{\max }=1000$, and the values $\left(I / \sigma_{Q U}\right)_{\text {crit }}>\left(I / \sigma_{Q U}\right)_{\max }$ (indicating the range in which input model values are not recoverable) are shaded in gray. Middle row: maximum recoverable index $\alpha_{\text {max }}$. The dotted gray line shows the 1:1 relation; values near or to the right of this line will not be accurately recoverable. Bottom row: fraction of data points above the critical S/N $\left(I / \sigma_{Q U}\right)_{\text {crit. }}$.

effect of uncertainty on the total intensity on the observed polarization fraction is negligible compared to that on the polarized intensity.

We find that the Ricean-mean model consistently performs better than single power-law fitting in accurately recovering $\alpha$ and $p_{\sigma_{Q U}}$. We therefore choose to apply this model to our observational data.

\section{An Example: JCMT POL-2 Observations of the L1688 Region of the Ophiuchus Molecular Cloud}

In order to test our model on real data, we used JCMT BISTRO survey (Ward-Thompson et al. 2017) $850 \mu \mathrm{m}$ POL-2 observations of three dense clumps within the Ophiuchus L1688 molecular cloud: Oph A, Oph B, and Oph C. These data sets were originally presented by Kwon et al. (2018), 


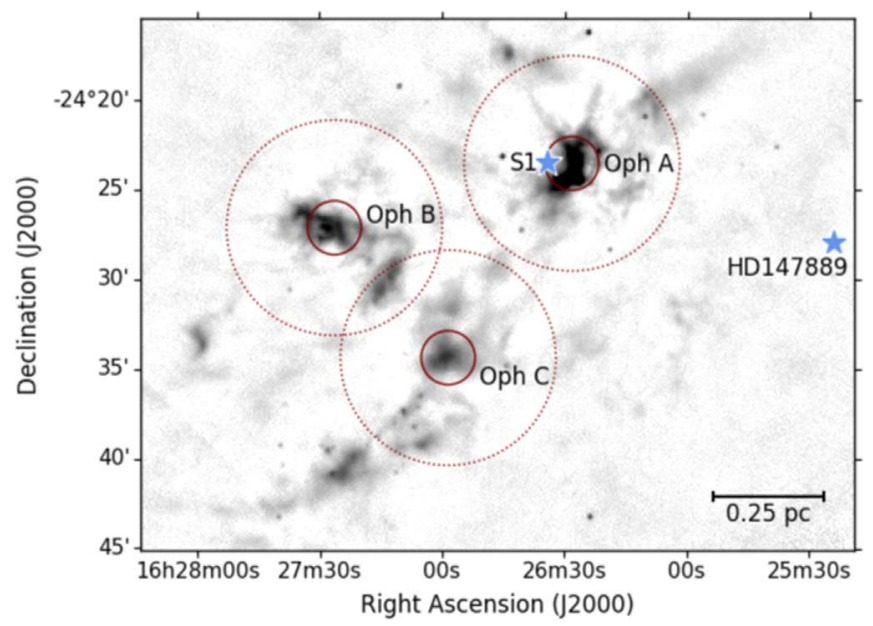

Figure 4. Finding chart for the L1688 region. The gray-scale image shows the JCMT Gould Belt Survey SCUBA-2 $850 \mu \mathrm{m}$ map of the region (Pattle et al. 2015). The three BISTRO fields are marked in red: solid lines show the central $3^{\prime}$ diameter regions with uniform noise characteristics used in this work, and dotted lines show the full extent of the observations. The locations of the B stars HD 147889 and S1 are marked with blue stars. A plane-of-sky distance of $0.25 \mathrm{pc}$ at our assumed distance to L1688 of $138 \mathrm{pc}$ is shown in the lower right corner.

Soam et al. (2018), and Liu et al. (2019), respectively, and were taken under JCMT project code M16AL004.

The Oph A and B clumps are active star formation sites containing outflow-driving protostellar sources and gravitationally bound prestellar cores, while Oph $\mathrm{C}$ has little or no ongoing star formation and contains only pressure-bound cores (Pattle et al. 2015). Oph B and $\mathrm{C}$ are cold ( $\sim 10 \mathrm{~K})$ clumps showing little sign of external influence. Oph $\mathrm{A}$ is warmer, $\sim 20 \mathrm{~K}$, and is in the vicinity of two B stars, HD 147889 (spectral type B2V, Wilking et al. 2008; effective temperature $T_{\text {eff }} \sim 20,800 \mathrm{~K}$, Silaj et al. 2010) and S1 (B4V, $T_{\text {eff }} \sim$ 17,000 K; Mookerjea et al. 2018). The immediate proximity of the latter of these significantly increases the ionizing photon flux on Oph A over that on other clumps in L1688 (Pattle et al. 2015). The geometry and radiation field of L1688 is discussed in detail by Liseau et al. (1999) and Stamatellos et al. (2007). A finding chart for the L1688 region is shown in Figure 4.

\subsection{Observations and Data Reduction}

We reduced each set of observations ${ }^{20}$ (20 observations of Oph A and C; 19 observations of Oph B) using the pol $2 m_{a p}{ }^{21}$ routine recently added to SMURF (Berry et al. 2005; Chapin et al. 2013) and the "2018 January" instrumental polarization model (Friberg et al. 2018). The data reduction process is as described by Soam et al. (2018), with the following modifications: (1) in the second stage of the data reduction process, the skyloop ${ }^{22}$ routine was used, in which each iteration of the mapmaker is performed on each of the observations in the set in turn, rather than each observation being reduced consecutively, as is the standard method; (2) variances in the final coadded maps were calculated according to the standard deviation of the measured values in each pixel across the 20

\footnotetext{
${ }^{20}$ A single JCMT BISTRO POL-2 observation consists of 40 minutes of observing time using the POL-2-DAISY scan pattern described by Friberg et al. (2016).

21 http://starlink.eao.hawaii.edu/docs/sc22.pdf

22 http://starlink.eao.hawaii.edu/docs/sc21.htx/sc21.html
}

observations, rather than as the mean of the rms of the bolometer counts in that pixel in each observation; and (3) in the final coadded maps, each observation was weighted according to the mean of its associated variance values. The net effect of these alterations is to improve homogeneity between observations and reduce noise in the final coadded maps. Based on the outcomes of fitting our Monte Carlo simulations, we did not attempt to debias the measured polarization fractions.

As discussed in Section 3, the Ricean model assumes that Stokes $Q$ and $U$ have uncorrelated uncertainties. This is a reasonable assumption for POL-2 data. The first step of the POL-2 data reduction process is to separate each bolometer time stream into $Q, U$, and $I$ time streams by fitting a sinusoidal function to the data using the calcqu ${ }^{23}$ routine. So long as the phase of the polarized emission (twice the position angle of the POL-2 half-wave plate) is accurately known at each point in the bolometer time stream, the $Q$ and $U$ time streams should be correctly separated. The $Q, U$, and $I$ data are thereafter reduced independently of one another.

In our model, we assume that the observed data can be accurately characterized by a single rms noise value, $\sigma_{Q U}$. The JCMT POL-2 observations use an observing mode wherein the central $3^{\prime}$ diameter region of each $12^{\prime}$ diameter observation has constant exposure time and so approximately constant rms noise (Friberg et al. 2016). We thus considered only this central region of each field. (In Oph B, this corresponds to the Oph B2 clump.) The three fields, along with their polarization vectors, are shown in Figure 5.

Each pixel in the output Stokes $Q$ and $U$ maps has associated variance values $V_{Q}$ and $V_{U}$, determined as described above. These variance maps can be converted into maps of $1 \sigma \mathrm{rms}$ noise by taking their square roots. We thus estimated a single rms noise value for our data,

$$
\left\langle\sigma_{Q U}\right\rangle=\frac{1}{2 N} \sum_{i=1}^{N}\left(\sqrt{V_{Q, i}}+\sqrt{V_{U, i}}\right)
$$

where $N$ is the number of pixels in the data set. We set $\sigma_{Q U}=\left\langle\sigma_{Q U}\right\rangle$ when performing model fitting. We retain the notation $\left\langle\sigma_{Q U}\right\rangle$ while fitting real data in order to emphasize that this is a measured property of the data set. The $\left\langle\sigma_{Q U}\right\rangle$ values measured in each field are listed in Table 1. We also calculated the uncertainties on the polarization fraction for each pixel from the variance values $V_{Q}, V_{U}$, and $V_{I}$ using Equation (19).

The JCMT POL-2 data are by default reduced onto $4^{\prime \prime}$ pixels. In order to investigate the dependence of the observed polarization fraction on rms noise, we also gridded the data to $4 n^{\prime \prime}$ pixels, where $n=1-8$, thereby generally reducing the rms noise, as shown in Table 1 . We note that binning to larger pixel sizes increases the chance of beam-averaging-related depolarization. However, for the $8^{\prime \prime}$ and $12^{\prime \prime}$ cases, as we grid from a pixel size of $\sim 1 / 3$ of the $850 \mu \mathrm{m}$ JCMT primary beam to slightly smaller than beam-sized pixels (the JCMT has an effective beam size of 14 !" 1 at $850 \mu \mathrm{m}$; Dempsey et al. 2013), we do not expect this to be an issue in at least these cases. We did not grid to larger pixel sizes than $32^{\prime \prime}$, as at larger pixel sizes, the rms noise values ceased to improve, and the number of data points became prohibitively small.

\footnotetext{
${ }^{23}$ http://starlink.eao.hawaii.edu/docs/sun258.htx/sun258ss5.html
} 

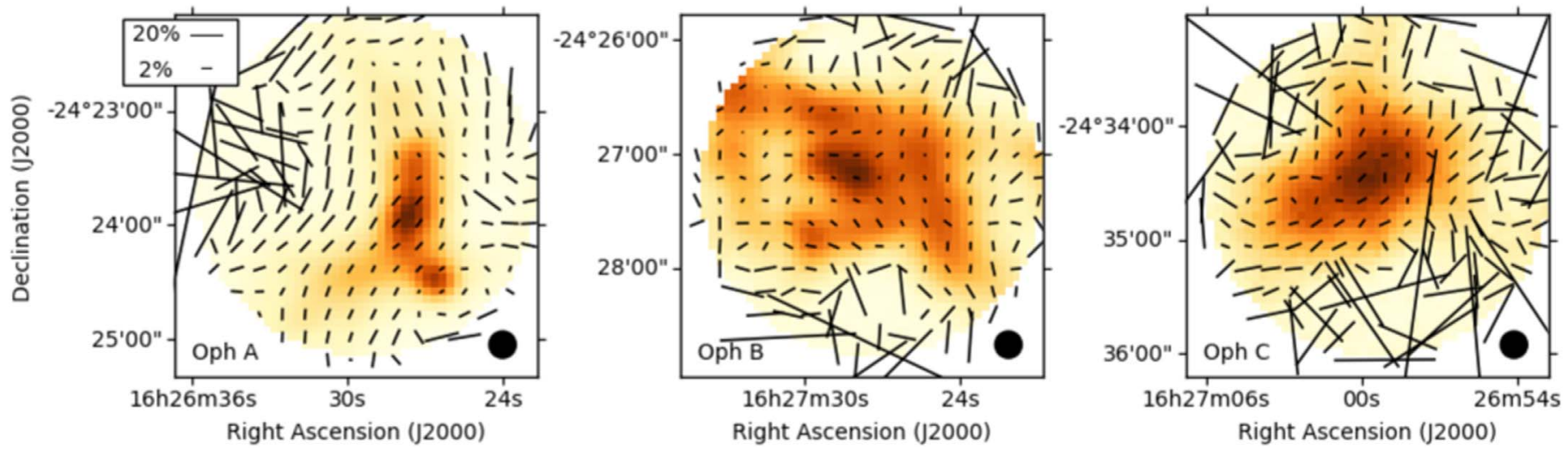

Figure 5. The POL-2 $850 \mu \mathrm{m}$ total intensity maps of the central $3^{\prime}$ of the BISTRO Oph A, B, and C fields, with polarization vectors overlaid. Vectors are sampled to a $12^{\prime \prime}$ grid and scaled according to $\sqrt{p^{\prime}}$. The polarization fractions have not been debiased. The $20 \%$ and $2 \%$ polarization vectors are shown in the upper left corner of the left panel, demonstrating the nonlinearity of the vector scale. The JCMT $850 \mu \mathrm{m}$ beam size is shown in the lower right corner of each plot.

\subsection{Fitting}

We fitted each data set with the mean of the Rice distribution (Equation (4)) using all data points and uncertainties calculated using Equation (19). Fitting was performed as described in Section 4.2. The models were normalized to the mean uncertainty in $Q$ and $U,\left\langle\sigma_{Q U}\right\rangle$, and the data were fitted for $p_{\sigma_{Q U}}$ and $\alpha$.

The results of our fitting are listed in Table 1 and plotted in Figures 6 and 7. It can be seen that in all cases, the data are well characterized by $\left\langle\sigma_{Q U}\right\rangle$ and well described by the Rice distribution, following Equation (4).

Table 1 lists the reduced $\chi^{2}$ values for each model and the null hypothesis. Under the null hypothesis, $p_{\sigma_{Q U}}=0$, so $p^{\prime}=\left(I /\left\langle\sigma_{Q U}\right\rangle\right)^{-1} \sqrt{\pi / 2}$ for all $I$. As in Section 4.2, we note that the uncertainties on $\delta p$ can be treated as Gaussian only at high $\mathrm{S} / \mathrm{N}$. The reduced- $\chi^{2}$ statistic is thus not strictly a direct comparison between the difference between the data and the model and the variance in the data. We therefore compare only the relative size of the reduced- $\chi^{2}$ statistics for the best-fit and null-hypothesis models, treating a smaller value of the reduced $\chi^{2}$ as being broadly representative of better agreement between the data and the model.

\subsection{Oph A}

Oph A shows clear evidence for a power-law behavior shallower than $\alpha=1$. Examination of Figure 6 shows significant deviation from $\alpha=1$ on $12^{\prime \prime}$ pixels. This is confirmed by the fitted models producing a reduced- $\chi^{2}$ statistic approximately three to four times smaller than that of the null hypothesis on all pixel sizes.

When fitting the mean of the Rice distribution to the Oph A data, we consistently recovered $p_{100 \mathrm{mJy}_{\text {beam }}{ }^{-1}} \approx 0.047$ and $\alpha \approx 0.34$. The results returned from $4^{\prime \prime}-28^{\prime \prime}$ pixel data are consistent within the fitting uncertainties, with no obvious signs of depolarization due to beam averaging, although the fitting results become more uncertain as the pixel size increases, as shown in Figure 8. Fitting of the Oph A data fails for $32^{\prime \prime}$ pixels; this is likely due to the relatively small number of remaining data points and the significant intrinsic scatter in the data around the best-fit model. In all cases, the best-fitting models produce reduced- $\chi^{2}$ statistics significantly greater than unity, suggesting more variation in the data than can be explained by our simple model alone.

We thus interpret our results as indicating that the Oph A data can be represented by the model $p_{100 \mathrm{mJy}_{\text {beam }}{ }^{-1}}=0.047$ and $\alpha=0.34$. Equations (11) and (14) suggest that, when $\sigma_{Q U} \approx 1 \mathrm{mJy}$ beam $^{-1}$, this behavior will be recoverable only when a significant number of data points fall above $\left(I / \sigma_{Q U}\right)_{\text {crit }} \sim 18$.

\subsection{Oph B}

In Oph B, the incompatibility of the data with the null hypothesis becomes more apparent with increasing pixel size. In the $4^{\prime \prime}$ case, the Oph B data do not clearly support a powerlaw index distinct from $\alpha=1$ being observed, with the Riceanmean model and the null hypothesis producing similar reduced- $\chi^{2}$ values.

In the $12^{\prime \prime}$ case, the data appear to be skewed above the nullhypothesis line in Figure 6. The Ricean-mean fitting results produce reduced- $\chi^{2}$ values a factor of $\sim 2$ smaller than that of the null hypothesis. Gridding the data to larger pixel sizes results in progressively smaller values of $p_{\sigma_{Q U}}$ and $\alpha$ being recovered, as shown in Figure 8. The $32^{\prime \prime}$ case is shown in Figure 7 and clearly shows that the data are not consistent with an index of $\alpha=1$.

The most representative values of $\alpha$ and $p_{100 \mathrm{mJy}_{\text {beam }}^{-1}}$ in Oph B are not very well constrained. To a certain extent, Figure 8 shows a stabilization in the fitted values of $\alpha$ and $p_{100 \mathrm{mJy}_{\text {beam }}^{-1}}$ in Oph B at lower rms noise values. On the larger pixel sizes considered, $\alpha \sim 0.6-0.7$. On $20^{\prime \prime}$ pixels and larger, $p_{100 \mathrm{mJy}_{\text {beam }}^{-1}}$ becomes consistent with a value of $\sim 0.02$. It is also possible that gridding to larger pixel sizes alters the observed average grain properties in Oph B, although, as discussed above, this does not appear to be the case in Oph A. Equations (11) and (14) suggest that $\alpha=0.65$ and $p_{100 \mathrm{mJy}_{\text {beam }}-1}=0.02$ would, for $\sigma_{Q U} \approx 1 \mathrm{mJy} \mathrm{beam}^{-1}$, be recoverable only when a significant number of data points fall above $\left(I / \sigma_{Q U}\right)_{\text {crit }} \sim 26$.

\subsection{Oph C}

We find that Oph $\mathrm{C}$ behaves similarly to Oph B. Due to its lower peak brightness than Oph B, the Oph $\mathrm{C}$ data show little evidence for deviation from an $I^{-1}$ behavior on beam-sized or smaller pixels. In the $4^{\prime \prime}$ and $8^{\prime \prime}$ data, the null hypothesis 
Table 1

Mean rms Noise in Stokes $Q$ and $U,\left\langle\sigma_{Q U}\right\rangle$; Number of Pixels, $N$; and Fitting Results for the Ricean-mean Model for L1688 Oph A, B, and C Data on Pixel Sizes of $4^{\prime \prime}-32^{\prime \prime}$

\begin{tabular}{|c|c|c|c|c|c|c|c|}
\hline \multirow{2}{*}{$\begin{array}{l}\text { Pixel } \\
\text { Size } \\
(\operatorname{arcsec})\end{array}$} & \multirow[b]{2}{*}{$\begin{array}{c}\left\langle\sigma_{Q U}\right\rangle \\
\left(\mathrm{mJy} \text { beam }^{-1}\right)\end{array}$} & \multirow[b]{2}{*}{$N$} & \multirow{2}{*}{$\begin{array}{l}\text { Null } \\
\frac{\chi^{2}}{N}\end{array}$} & \multicolumn{4}{|c|}{ Ricean-mean Model } \\
\hline & & & & $\alpha$ & $p_{\sigma_{Q U}}$ & $p_{100 \mathrm{mJy}}$ beam $^{-1}$ & $\frac{\chi^{2}}{N-2}$ \\
\hline \multicolumn{8}{|c|}{ Oph A } \\
\hline 4 & $3.29 \pm 0.55$ & 1456 & 33.3 & $0.34 \pm 0.02$ & $0.15 \pm 0.02$ & $0.047 \pm 0.009$ & 9.2 \\
\hline 8 & $1.88 \pm 0.36$ & 400 & 101.0 & $0.34 \pm 0.03$ & $0.18 \pm 0.04$ & $0.047 \pm 0.016$ & 24.9 \\
\hline 12 & $1.45 \pm 0.50$ & 192 & 189.4 & $0.33 \pm 0.05$ & $0.19 \pm 0.06$ & $0.047 \pm 0.025$ & 45.1 \\
\hline 16 & $1.15 \pm 0.44$ & 114 & 291.9 & $0.34 \pm 0.06$ & $0.21 \pm 0.09$ & $0.046 \pm 0.032$ & 73.0 \\
\hline 20 & $1.07 \pm 0.66$ & 79 & 397.2 & $0.39 \pm 0.08$ & $0.31 \pm 0.18$ & $0.053 \pm 0.050$ & 110.6 \\
\hline 24 & $0.95 \pm 0.66$ & 56 & 437.1 & $0.38 \pm 0.10$ & $0.30 \pm 0.20$ & $0.051 \pm 0.058$ & 98.9 \\
\hline 28 & $1.02 \pm 0.77$ & 47 & 480.4 & $0.31 \pm 0.12$ & $0.18 \pm 0.16$ & $0.043 \pm 0.062$ & 115.5 \\
\hline 32 & $0.60 \pm 0.16$ & 31 & 832.0 & $0.00 \pm 0.16$ & $0.02 \pm 0.03$ & $0.020 \pm 0.046$ & 172.1 \\
\hline \multicolumn{8}{|c|}{ Oph B } \\
\hline 4 & $3.37 \pm 0.57$ & 1404 & 1.4 & $0.86 \pm 0.03$ & $0.89 \pm 0.11$ & $0.048 \pm 0.011$ & 1.0 \\
\hline 8 & $1.93 \pm 0.41$ & 390 & 2.5 & $0.78 \pm 0.05$ & $0.73 \pm 0.18$ & $0.034 \pm 0.015$ & 1.4 \\
\hline 12 & $1.49 \pm 0.54$ & 191 & 3.7 & $0.76 \pm 0.07$ & $0.68 \pm 0.25$ & $0.028 \pm 0.018$ & 2.0 \\
\hline 16 & $1.18 \pm 0.49$ & 115 & 4.7 & $0.70 \pm 0.09$ & $0.53 \pm 0.26$ & $0.024 \pm 0.021$ & 2.2 \\
\hline 20 & $1.12 \pm 0.76$ & 78 & 6.6 & $0.66 \pm 0.12$ & $0.44 \pm 0.29$ & $0.023 \pm 0.027$ & 3.2 \\
\hline 24 & $0.99 \pm 0.73$ & 58 & 7.7 & $0.71 \pm 0.12$ & $0.58 \pm 0.38$ & $0.022 \pm 0.026$ & 3.5 \\
\hline 28 & $1.03 \pm 0.77$ & 47 & 6.4 & $0.57 \pm 0.18$ & $0.22 \pm 0.22$ & $0.016 \pm 0.030$ & 3.2 \\
\hline 32 & $0.61 \pm 0.16$ & 32 & 12.2 & $0.59 \pm 0.16$ & $0.34 \pm 0.34$ & $0.017 \pm 0.030$ & 4.2 \\
\hline \multicolumn{8}{|c|}{ Oph C } \\
\hline 4 & $3.70 \pm 0.65$ & 1239 & 0.9 & $0.83 \pm 0.03$ & $0.75 \pm 0.09$ & $0.049 \pm 0.011$ & 0.7 \\
\hline 8 & $2.12 \pm 0.45$ & 359 & 1.4 & $0.84 \pm 0.04$ & $0.93 \pm 0.16$ & $0.037 \pm 0.012$ & 1.0 \\
\hline 12 & $1.64 \pm 0.63$ & 177 & 2.1 & $0.76 \pm 0.07$ & $0.70 \pm 0.20$ & $0.031 \pm 0.018$ & 1.3 \\
\hline 16 & $1.30 \pm 0.54$ & 109 & 2.3 & $0.75 \pm 0.07$ & $0.72 \pm 0.24$ & $0.027 \pm 0.018$ & 1.3 \\
\hline 20 & $1.22 \pm 0.80$ & 75 & 2.6 & $0.69 \pm 0.09$ & $0.52 \pm 0.22$ & $0.025 \pm 0.020$ & 1.3 \\
\hline 24 & $1.10 \pm 0.87$ & 55 & 2.9 & $0.71 \pm 0.12$ & $0.52 \pm 0.29$ & $0.021 \pm 0.023$ & 1.7 \\
\hline 28 & $1.15 \pm 0.88$ & 45 & 4.0 & $0.58 \pm 0.14$ & $0.30 \pm 0.21$ & $0.023 \pm 0.030$ & 2.0 \\
\hline 32 & $0.67 \pm 0.18$ & 32 & 6.0 & $0.63 \pm 0.11$ & $0.52 \pm 0.30$ & $0.022 \pm 0.025$ & 2.0 \\
\hline
\end{tabular}

Note. Note that the results for Oph A on $32^{\prime \prime}$ pixels are not reliable.

produces a reduced- $\chi^{2}$ value comparable to that of the Riceanmean model, and there is no clear evidence that the Riceanmean model provides a better description of the data. In the $12^{\prime \prime}$ case, shown in Figure 6, there is only a marginal improvement in goodness of fit over the null-hypothesis case.

As in Oph B, gridding to larger pixels produces smaller values of both $\alpha$ and $p_{100 \mathrm{mJy}_{\text {beam }}-1}$ and makes the deviation of the data from the null-hypothesis behavior more apparent. Figure 7 qualitatively shows the similarity between the Oph B and $\mathrm{C}$ data, while Figure 8 shows that the fitting results of Oph B and C agree very closely in all cases.

\subsection{Discussion}

Our results suggest that grains in Oph A are intrinsically better aligned with the magnetic field than those in Oph $\mathrm{B}$ and C. Grains in Oph B and C appear to lose what alignment they have with the magnetic field more precipitously with increasing density (or extinction) than is the case in Oph A. Oph B and C appear to have indistinguishable grain properties despite their differing star formation histories, while Oph A and B behave significantly differently despite having comparable mass and both being active sites of star formation.

We estimated the upper-limit ionizing fluxes on Oph A, B, and C from the B stars HD 147889 and S1 as a qualitative indicator of the differences in interstellar radiation field (ISRF) on the three clumps. We took the flux of Lyman continuum photons to be $\sim 10^{20.4} \mathrm{~cm}^{-2} \mathrm{~s}^{-1}$ from the surface of HD 147889 and $\sim 10^{18.5} \mathrm{~cm}^{-2} \mathrm{~s}^{-1}$ from the surface of S1 (Pattle et al. 2015, and references therein). We determined plane-of-sky distances from HD 147889 and S1 to the centers of Oph A, B, and C, assuming a distance to L1688 of $138 \mathrm{pc}$ (Ortiz-León et al. 2018), and so estimated upper-limit ionizing fluxes from the stars on each region. ${ }^{24}$ These fluxes are listed in Table 2. We assume that the global ISRF on the three regions is comparable (a reasonable assumption, given the clumps' proximity to one another). It can be seen that the ionizing flux on Oph A is an order of magnitude larger than that on Oph B and $\mathrm{C}$, and that this difference is primarily caused by the proximity of S1 to Oph A. It is likely that HD 147889 will affect the three clumps similarly, while S1's influence is dominant in Oph A but negligible elsewhere.

\footnotetext{
${ }^{24}$ We note that if we adopted the three-dimensional model of L1688 proposed by Liseau et al. (1999), the distance from HD 147889 to the three clumps would be $0.86 \mathrm{pc}$ to Oph A, $1.33 \mathrm{pc}$ to Oph B, and $1.03 \mathrm{pc}$ to Oph C (distances scaled to account for their assumed distance to L1688 of $150 \mathrm{pc}$ ). This would alter our inferred fluxes from HD 147889 by factors of $\sim 0.5, \sim 0.6$, and $\sim 0.8$ in Oph A, B, and C respectively. This would not change the conclusions of our analysis.
} 

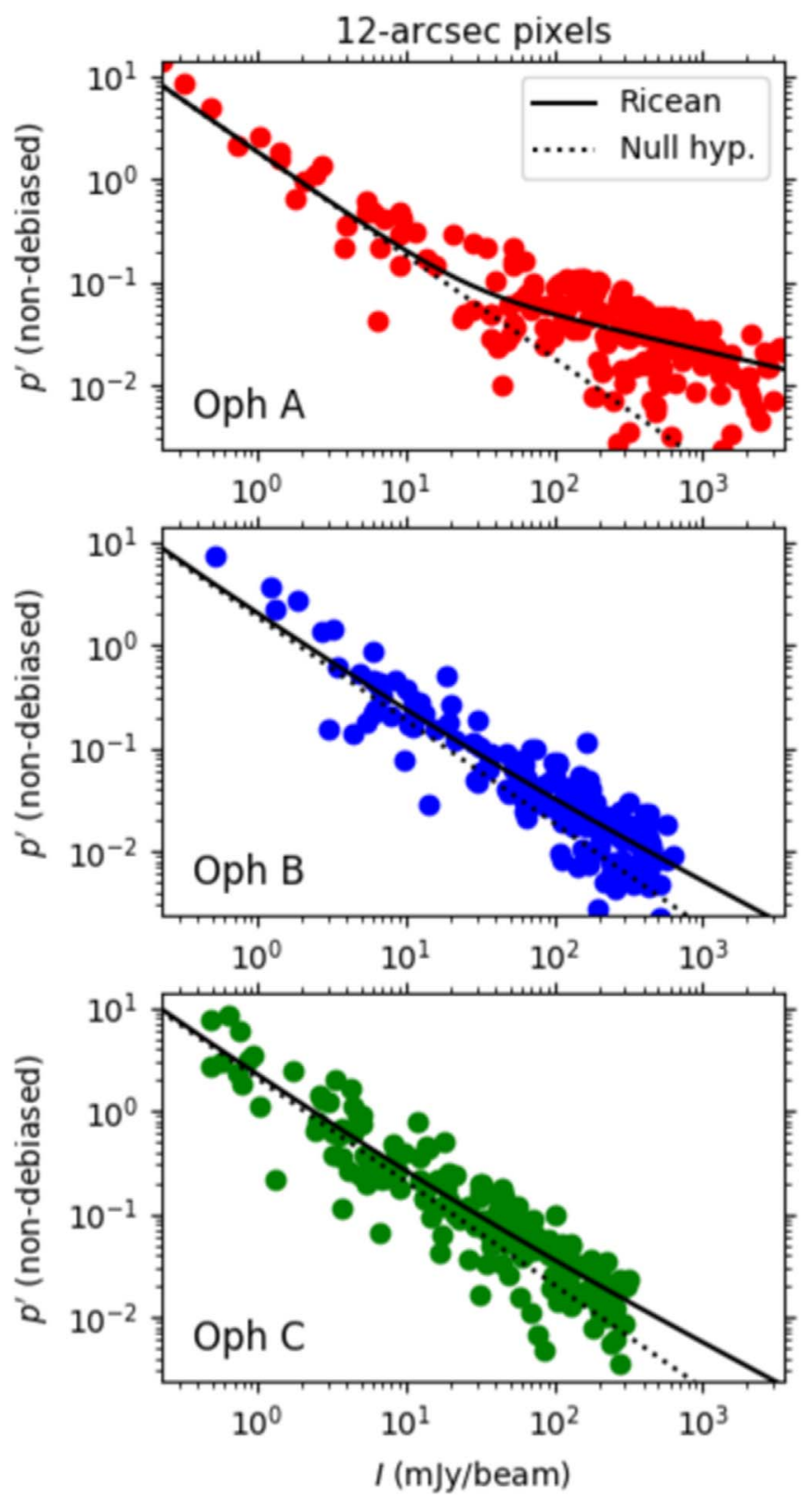

Figure 6. Plot of observed polarization fraction $p^{\prime}$ vs. total intensity $I$ for the Oph A, B, and $\mathrm{C}$ data, gridded onto $12^{\prime \prime}$ pixels. Top row: Oph A; middle row: Oph B; bottom row: Oph C. The solid black line shows the best-fitting Riceanmean model. The dotted black line shows the expected behavior under the null hypothesis. Note that the polarization fractions have not been debiased.

The most likely explanation for the better grain alignment in Oph A is thus the elevated photon flux on that region, primarily resulting from the proximity of the star $S 1$. Under the radiative torque alignment (RAT) paradigm of grain alignment (Lazarian \& Hoang 2007; Andersson et al. 2015), this stronger and bluer radiation field on Oph A would allow grain alignment to persist to a higher optical depth. The strongly anisotropic radiation field on Oph A might also favor better grain alignment in this region (Dolginov \& Mitrofanov 1976; Onaka 2000; Weingartner \& Draine 2003). We note that the difference in behavior between Oph A and Oph B, both of which are actively forming stars, suggests that the better grain alignment in Oph A is primarily driven by external influence, not by short-wavelength flux from protostellar sources within the clump.

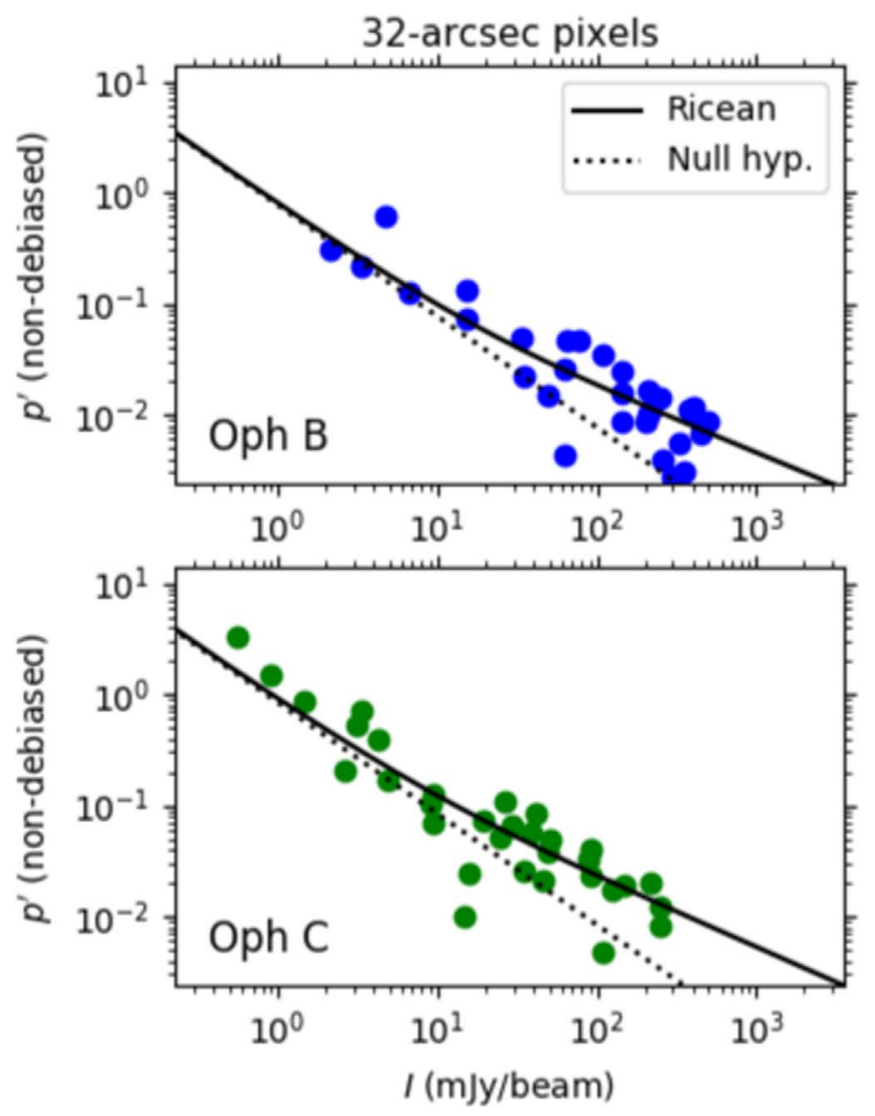

Figure 7. Observed polarization fraction $p^{\prime}$ vs. total intensity $I$ for the B and C data, with data gridded to $32^{\prime \prime}$ pixels. The polarization fractions have not been debiased. Best-fitting models are shown as in Figure 6. Note that both data sets show significant deviation from the null-hypothesis behavior.

We note that the ionizing flux from these stars will not itself directly contribute to grain alignment within the clumps. In order to drive RAT grain alignment, the wavelength of the incident radiation must be shorter than twice the size of the largest grains, i.e., $\lesssim 1-2 \mu \mathrm{m}$ (Andersson et al. 2015). The short-wavelength photons considered here will undergo multiple scatterings before they can contribute significantly to grain alignment, while longer-wavelength emission from the stars may contribute more directly, particularly in Oph A, thanks to its proximity to $\mathrm{S} 1$. We emphasize that the calculations above only qualitatively demonstrate the global elevation of photon flux on Oph A over the other two clumps. Modeling of the detailed radiation field in L1688 is beyond the scope of this work.

The magnetic field in Oph A may also be intrinsically more ordered in Oph A than in Oph B and C, as the clump's location between HD 147889 and S1 could result in its molecular gas, and thus its magnetic field, being compressed by the HD 147889 photon-dominated region (PDR) and the $\mathrm{S} 1$ reflection nebula. In contrast, Oph $\mathrm{B}$ and $\mathrm{C}$ are evolving in relative isolation from the two $\mathrm{B}$ stars and are not undergoing significant compression. In this case, the lower value of $\alpha$ in Oph A might in part result from its more ordered internal magnetic field, with less vector cancellation of the observed polarization fraction along the line of sight occurring in Oph A than in the other two regions.

Another possible cause of better grain alignment in Oph A than in the other two clumps is grain growth in the dense 

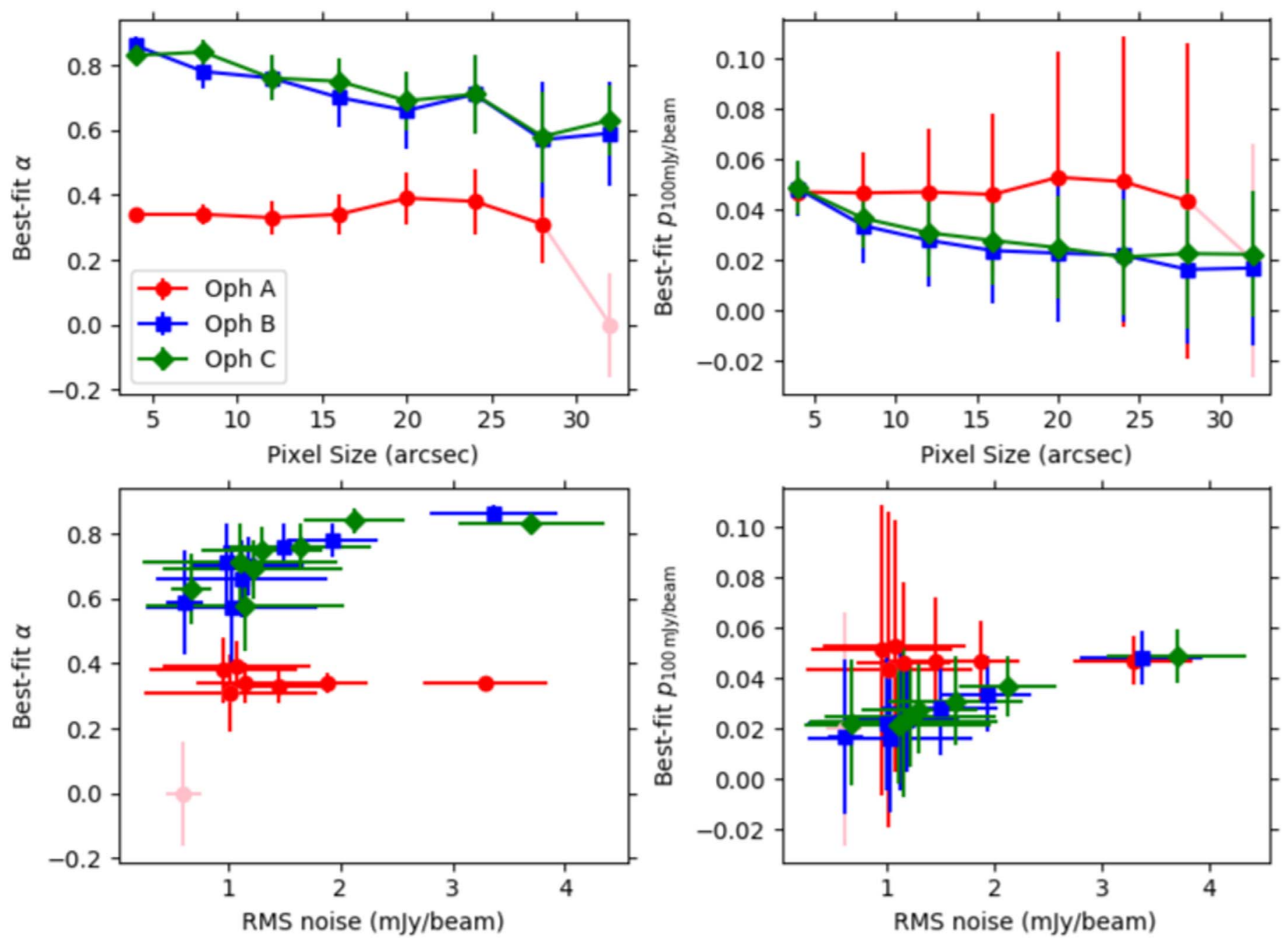

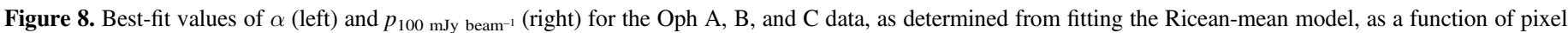
size (top) and rms noise $\left\langle\sigma_{Q U}\right\rangle$ (bottom). Fitting results for Oph A on 32" pixels are shown in pink, as this result is not reliable.

Table 2

Ionizing Flux on Oph A, B, and C from the Stars HD 147889 and S1

\begin{tabular}{lcclccc}
\hline \hline & \multicolumn{2}{c}{$\begin{array}{c}\text { Plane-of-sky Dis- } \\
\text { tance }(\mathrm{pc})\end{array}$} & & \multicolumn{3}{c}{$\begin{array}{c}\text { Upper-limit Ionizing Photon Flux } \\
\left(\mathrm{s}^{-1} \mathrm{~m}^{-2}\right)\end{array}$} \\
\cline { 2 - 3 } \cline { 5 - 7 } Region & HD 147889 & S1 & & HD 147889 & S1 & Total \\
\hline Oph A & 0.62 & 0.05 & & $7.0 \times 10^{10}$ & $1.5 \times 10^{11}$ & $2.2 \times 10^{11}$ \\
Oph B & 1.13 & 0.51 & & $2.1 \times 10^{10}$ & $1.2 \times 10^{9}$ & $2.2 \times 10^{10}$ \\
Oph C & 0.91 & 0.50 & & $3.2 \times 10^{10}$ & $1.2 \times 10^{9}$ & $3.4 \times 10^{10}$ \\
\hline
\end{tabular}

regions of Oph A. The peak gas density of Oph A is approximately 1 order of magnitude higher than in Oph B and C (Motte et al. 1998). Such high densities might provide the necessary conditions for the formation of large dust grains (e.g., Hirashita \& Li 2013). In the RAT paradigm, larger dust grains can be aligned by longer-wavelength photons, as described above; thus, the presence of large grains would allow grain alignment to persist to a higher optical depth.

While our results support better grain alignment in Oph A than in the other clumps, they do not suggest that grains in Oph $\mathrm{B}$ and $\mathrm{C}$ have no alignment with the magnetic field. Our modeling suggests that an index $\alpha \sim 0.6-0.7$ is plausible for both Oph B and C, suggesting that some degree of grain alignment may persist to high optical depths within these clumps.

These results suggest that grain alignment could persist to significantly higher densities within starless clumps and cores than has previously been believed to be the case (e.g., Jones et al. 2015; Kwon et al. 2018; Soam et al. 2018), even in the absence of a short-wavelength illuminating source. This is consistent with recent modeling results, which suggest that grains remain well aligned with the magnetic field at gas densities $>10^{3} \mathrm{~cm}^{-3}$ (Seifried et al. 2019).

\subsection{Limitations of the Fitting Process}

The simple model we consider in this work is subject to a number of limitations.

We emphasize that in this work, we selected our data such that they can be well characterized by a single rms noise value in both Stokes $Q$ and $U$. Data sets containing significant variation in rms noise would produce additional vertical spread in the $p^{\prime}-I$ plane, further complicating the recovery of an accurate value of $\alpha$.

Our data show scatter about the best-fit line greater than can be explained by instrumental uncertainty alone, particularly in Oph A, which in all cases shows reduced- $\chi^{2}$ values significantly larger than those in Oph B and $\mathrm{C}$, and where 
fitting fails for the largest pixel size considered here, likely due to significant intrinsic scatter in the data and the small number of data points to which the model can be fitted. In order to demonstrate the statistical properties of the data, we have chosen a very simple model in which the data are characterized by a single power law. More complex relationships between the polarization fraction and intensity could be investigated in future studies, as well as accounting for intrinsic variation in $p_{\sigma_{Q U}}$ and $\alpha$ within a given region.

Our model is unphysical in that it suggests that the polarization fraction can increase indefinitely at small $I$. We are implicitly assuming first that there is a turnover in behavior at a density below which the polarization fraction becomes a shallower function of intensity, tending to the value in the lowdensity ISM. Second, we assume that this turnover occurs at densities lower than we can probe with POL-2. (In approximately isothermal environments, the SCUBA-2 camera is effectively volume-density-limited in its detections; see WardThompson et al. 2016.)

Our results suggest that an observed index of $\alpha \simeq 1$ in the submillimeter data (e.g., Alves et al. 2014; Jones et al. 2015; Liu et al. 2019) is not sufficient to claim that nonaligned grains have been observed. However, if a break or turnover in behavior from a shallow power law $(\alpha<1)$ to an index of $\alpha=1$ with increasing intensity were observed within a single polarized submillimeter emission data set, this would be strong evidence for the loss of grain alignment at high intensities. This is because if an index $\alpha<1$ were recoverable at intermediate intensities, then an $\alpha=1$ index seen at high intensities in the same data set could not simply result from having insufficient $\mathrm{S} / \mathrm{N}$ to measure a shallower index and would thus be indicative of a genuine change in behavior with intensity. We do not see any evidence of such a break in behavior in Ophiuchus.

\subsection{Relation between $\mathrm{I}$ and Visual Extinction $\mathrm{A}_{\mathrm{V}}$}

In this paper, we consider only the relationship between $p$ and $I$, with the goal of accurately determining their underlying relationship in the presence of Ricean noise. However, it is important to emphasize that the true physical relationship under investigation is not between $p$ and $I$ but between $p$ and $A_{V}$.

Recent POL-2 studies (Juvela et al. 2018; Coudé et al. 2019; Wang et al. 2019) show a shallower relationship between $p^{\prime}$ and Herschel Space Observatory-derived dust opacity/optical depth measurements (a proxy for $A_{V}$, as discussed below) than between $p^{\prime}$ and $I$. We here present a simple argument for why we expect the $p-A_{V}$ relationship to be intrinsically shallower than the $p-I$ relationship.

The submillimeter intensity $I_{\nu}$ of thermal dust emission at a frequency $\nu$ is given by

$$
I_{\nu}=B_{\nu}(T) \tau_{\nu} \Omega
$$

where $B_{\nu}(T)$ is the Planck function at temperature $T, \tau_{\nu}$ is the submillimeter optical depth, and $\Omega$ is the solid angle (e.g., Hildebrand 1983). We henceforth assume that we are observing over a constant area, so

$$
I_{\nu} \propto B_{\nu}(T) \tau_{\nu}
$$

Assuming $\tau_{\nu} \propto A_{V}$, i.e., optically thin submillimeter emission (e.g., Jones et al. 2015) and constant dust optical properties along the line of sight,

$$
I_{\nu} \propto B_{\nu}(T) A_{V} .
$$

Thus, $I$ is a direct tracer of $A_{V}$ only where $T$ is constant. However, in most environments in molecular clouds, $B_{\nu}(T)$ and $I$ are observed to be anticorrelated (e.g., Kirk et al. 2013; Könyves et al. 2015). This is a physical effect, with cooling being caused by self-shielding in dense environments (e.g., Glover \& Clark 2012). Such cooling is expected in regions that do not contain embedded massive stars causing internal heating, such as the dense clumps of L1688 (Stamatellos et al. 2007). We parameterize the relationship between $B_{\nu}(T)$ and $I_{\nu}$ as

$$
B_{\nu}(T) \propto I_{\nu}^{\gamma},
$$

initially placing no constraint on $\gamma$ (note that here $B_{\nu}(T)$ is the source function of the dust emission; see Equation (26)). Combining Equations (10), (27), and (28),

$$
p \propto A_{V}^{-\frac{\alpha}{1-\gamma}} \text {. }
$$

If $\gamma<0\left(B_{\nu}(T)\right.$, and so $T$, decreases with increasing $\left.I_{\nu}\right)$, then

$$
\frac{1}{1-\gamma}<1
$$

Thus, in most physical environments in molecular clouds, any power-law relationship between $A_{V}$ and $p$ must be shallower (and likely more weakly correlated) than that between $I$ and $p$. We note that $\gamma<0$ would not hold in the presence of significant heating by sources located at high $A_{V}$, but in that case, we might expect these heating sources to also be driving grain alignment in their vicinity.

This analysis further suggests that grains could be better aligned at higher extinction than has previously been believed.

The exact nature of the relationship between $I, T$, and $\tau$ is not obtainable from single-wavelength observations such as those considered here. However, forthcoming multiwavelength studies will allow more direct investigation of the $I-A_{V}$ relationship.

\section{Summary}

The dependence of the polarization fraction on total intensity in polarized submillimeter emission measurements is typically parameterized as a power law and used to infer the efficiency of dust grain alignment with the magnetic field in star-forming clouds and cores. In this work, we have demonstrated that significant $\mathrm{S} / \mathrm{N}$ and well-characterized noise properties are required to recover a genuine power-law relationship between the polarization fraction and total intensity.

We presented a simple model for the dependence of the polarization fraction on total intensity in molecular clouds and so demonstrated that below an $\mathrm{S} / \mathrm{N}$ threshold of $I / \sigma_{Q U}=\left(p_{\sigma_{Q U}}^{-1} \sqrt{\pi / 2}\right)^{\frac{1}{1-\alpha}}$, a power-law index of -1 will always be observed, as a result of the addition in quadrature of Stokes $Q$ and $U$ components and the approximate $1 / I$ dependence of the error in the polarization fraction. For power-law indices $\alpha<1$, the intrinsic dependence of the polarization fraction on intensity will be recoverable at high $\mathrm{S} / \mathrm{N}$. However, a genuine measurement of $p \propto I^{-1}$ —indicating unaligned grains-will be indistinguishable from statistical noise in most, if not all, physically realistic scenarios without additional information. We demonstrated that fitting a single power law is likely to result in overestimation of $\alpha$ and so of the degree of depolarization occurring. We further found that fitting the 
mean of the Rice distribution to non-debiased data will accurately recover both $p_{\sigma_{Q U}}$ and $\alpha$, provided a reasonable number of data points fall above the required $\mathrm{S} / \mathrm{N}$ threshold.

We used JCMT POL-2 observations of three clumps in the L1688 region of the Ophiuchus molecular cloud to demonstrate the statistical behavior described above. We found that the Oph A region, which is illuminated by two B stars, shows significantly better grain alignment than the neighboring Oph $\mathrm{B}$ and $\mathrm{C}$. We found a power-law index of $\alpha \approx 0.34$ in Oph A, significantly shallower than that found by previous works. The power-law indices in Oph B and C are less well constrained but steeper than that of Oph A, and they are likely to be in the range $\alpha \sim 0.6-0.7$. Oph $\mathrm{B}$ and $\mathrm{C}$ have intrinsically lower polarization fractions than Oph A at a total intensity of $100 \mathrm{mJy}$ beam $^{-1}$, with emission from Oph A being $4.7 \%$ polarized, while emission from Oph B and C is $\sim 2 \%$ polarized. Oph C, a quiescent cloud, appears to behave comparably to the actively star-forming Oph B. Our results thus suggest that grain alignment in Ophiuchus is driven by the external radiation field on the clumps, not by internal radiation sources.

These results suggest that grain alignment could persist to significantly higher densities within starless clumps and cores than has previously been believed to be the case. Submillimeter polarization measurements could thus potentially trace the magnetic field morphology in dense, star-forming gas.

K.P., S.P.L., and J.W.W. acknowledge support from the Ministry of Science and Technology (Taiwan) under grant No. 106-2119-M-007-021-MY3. K.P. was an International Research Fellow of the Japan Society for the Promotion of Science for part of the duration of this project. R.S.F. is supported by Japan Society for the Promotion of Science
(JSPS) Grants-in-Aid for Scientific Research, KAKENHI grant No. 19H01938. R.S.F and T.H. acknowledge the Multiwavelength Data Analysis System operated by the Astronomy Data Center (ADC), National Astronomical Observatory of Japan (NAOJ). W.K. and C.W.L were supported by the Basic Science Research Program through the National Research Foundation of Korea (NRF-2016R1C1B2013642 and 2019R1A2C1010851, respectively). The James Clerk Maxwell Telescope is operated by the East Asian Observatory on behalf of the National Astronomical Observatory of Japan, Academia Sinica Institute of Astronomy and Astrophysics, Korea Astronomy and Space Science Institute, and Center for Astronomical Mega-Science (as well as the National Key R\&D Program of China with No. 2017YFA0402700). Additional funding support is provided by the Science and Technology Facilities Council of the United Kingdom and participating universities in the United Kingdom and Canada. The authors wish to recognize and acknowledge the very significant cultural role and reverence that the summit of Maunakea has always had within the indigenous Hawaiian community. We are most fortunate to have the opportunity to conduct observations from this mountain.

Facility: James Clerk Maxwell Telescope (JCMT).

Software: Starlink (Currie et al. 2014), Astropy (Astropy Collaboration et al. 2013, 2018).

\section{Appendix \\ Appendix: List of Symbols Used in This Work}

Table 3 lists the symbols used in this work, along with their units and the section of the paper in which they are defined.

Table 3

Symbols Used in This Work

\begin{tabular}{|c|c|c|c|}
\hline Symbol & Definition & Units & Section Defined in \\
\hline$\alpha$ & Power-law index, $p \propto \Gamma^{\alpha}$ & Dimensionless & 1,3 \\
\hline$\alpha_{\max }$ & Steepest recoverable $\alpha$ for given $p_{\sigma_{Q U}}$ and $\left(I / \sigma_{Q U}\right)_{\max }$ & Dimensionless & 4.2 \\
\hline$I$ & Stokes $I$ intensity & Intensity & 2 \\
\hline$\sigma_{I}$ & rms noise in Stokes $I$ & Intensity & 2 \\
\hline$\delta I$ & Measurement uncertainty on Stokes $I$ & Intensity & 2 \\
\hline$I_{\mathrm{obs}}$ & Stokes I intensity in Monte Carlo model & Intensity & 4 \\
\hline$\Delta I$ & Measurement error on Stokes $I$ in Monte Carlo model & Intensity & 4 \\
\hline $\mathcal{I}_{0}$ & Modified Bessel function of order zero & $\cdots$ & 2.1 \\
\hline $\mathcal{I}_{1}$ & Modified Bessel function of order 1 & $\cdots$ & 2.1 \\
\hline $\mathcal{L}_{\frac{1}{2}}$ & Laguerre polynomial of order $\frac{1}{2}$ & $\ldots$ & 2.1 \\
\hline$N$ & Number of pixels in a given data set & Dimensionless & 5.1 \\
\hline$p$ & Intrinsic polarization fraction & Dimensionless & 2 \\
\hline$p^{\prime}$ & Measured polarization fraction & Dimensionless & 2.1 ; see also 4.2 \\
\hline$p_{\mathrm{db}}^{\prime}$ & Debiased measured polarization fraction & Dimensionless & 2.2 \\
\hline$p_{0}$ & Polarization fraction at reference intensity $I_{0}$ & Dimensionless & 3 \\
\hline$p_{\sigma_{Q U}}$ & Polarization fraction at reference intensity $\sigma_{Q U}$ & Dimensionless & 3 \\
\hline$p_{100 \text { mJy beam }}{ }^{-1}$ & Polarization fraction at $I=100 \mathrm{mJy} \mathrm{beam}^{-1}$ & Dimensionless & 3 \\
\hline$\sigma_{p}$ & rms noise in polarization fraction & Dimensionless & 2.1 \\
\hline$\delta p$ & Measurement uncertainty on polarization fraction & Dimensionless & 4.1 \\
\hline$\mu_{p}$ & Mean of Rice-distributed polarization fraction & Dimensionless & 2.1 \\
\hline$P$ & Intrinsic polarized intensity & Intensity & 2 \\
\hline$P^{\prime}$ & Measured polarized intensity & Intensity & 2.1 \\
\hline$P_{\mathrm{db}}^{\prime}$ & Debiased measured polarized intensity & Intensity & 2.2 \\
\hline$Q$ & Stokes $Q$ intensity & Intensity & 2 \\
\hline$\sigma_{Q}$ & rms noise in Stokes $Q$ & Intensity & 2 \\
\hline$\delta Q$ & Measurement uncertainty on Stokes $Q$ & Intensity & 2 \\
\hline
\end{tabular}


Table 3

(Continued)

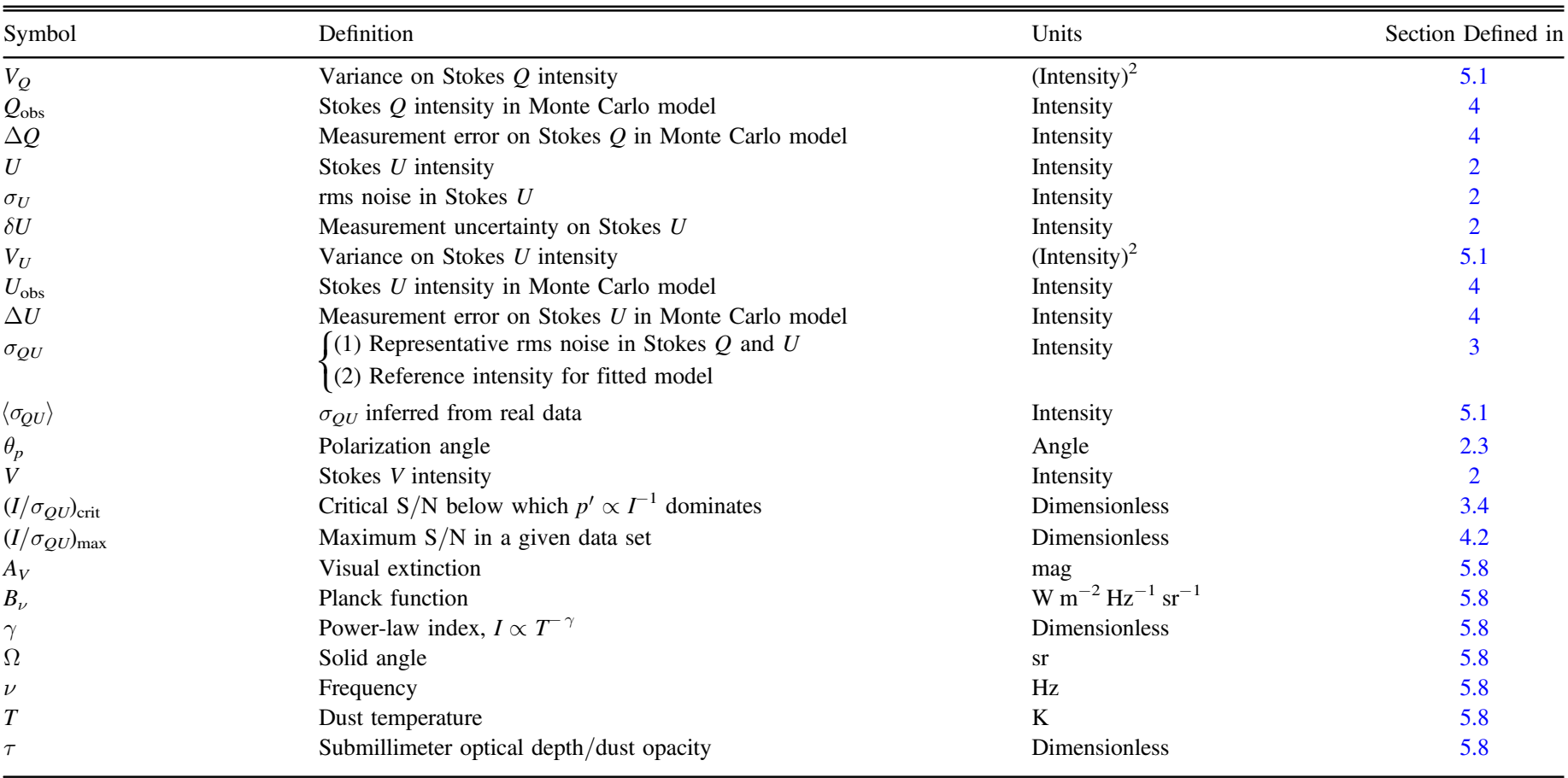

\section{ORCID iDs}

Kate Pattle (i) https://orcid.org/0000-0002-8557-3582 Shih-Ping Lai (i) https://orcid.org/0000-0001-5522-486X Tetsuo Hasegawa (i) https://orcid.org/0000-0003-1853-0184 Jia-Wei Wang (1) https://orcid.org/0000-0002-6668-974X Ray S. Furuya @ https://orcid.org/0000-0003-0646-8782 Derek Ward-Thompson (i) https://orcid.org/0000-00031140-2761

Pierre Bastien (iㅏ https://orcid.org/0000-0002-0794-3859

Simon Coudé (i) https://orcid.org/0000-0002-0859-0805 Chakali Eswaraiah (i) https://orcid.org/0000-0003-4761-6139

Lapo Fanciullo (1) https://orcid.org/0000-0001-9930-9240 James di Francesco (1) https://orcid.org/0000-0002-9289-2450 Thiem Hoang (i) https://orcid.org/0000-0003-2017-0982 Gwanjeong Kim (1) https://orcid.org/0000-0003-2011-8172 Woojin Kwon (1) https://orcid.org/0000-0003-4022-4132 Chang Won Lee $\mathbb{1}$ h https://orcid.org/0000-0002-3179-6334 Sheng-Yuan Liu 난 https://orcid.org/0000-0003-4603-7119 Tie Liu (ib https://orcid.org/0000-0002-5286-2564 Masafumi Matsumura (ib https://orcid.org/0000-00026906-0103

Takashi Onaka (1) https://orcid.org/0000-0002-8234-6747 Sarah Sadavoy iㅜ https://orcid.org/0000-0001-7474-6874 Archana Soam (i) https://orcid.org/0000-0002-6386-2906

\section{References}

Abdi, A., Tepedelenlioglu, C., Kaveh, M., \& Giannakis, G. 2001, IEEE Commun. Lett, 5, 92

Alves, F. O., Frau, P., Girart, J. M., et al. 2014, A\&A, 569, L1

Alves, F. O., Frau, P., Girart, J. M., et al. 2015, A\&A, 574, C4

Andersson, B.-G., Lazarian, A., \& Vaillancourt, J. E. 2015, ARA\&A, 53, 501

Astropy Collaboration, Price-Whelan, A. M., Sipőcz, B. M., et al. 2018, AJ, 156,123
Astropy Collaboration, Robitaille, T. P., Tollerud, E. J., et al. 2013, A\&A, 558, A33

Bastien, P., Vernet, E., Drissen, L., et al. 2007, in ASP Conf. Ser. 364, The Future of Photometric, Spectrophotometric and Polarimetric Standardization, ed. C. Sterken (San Francisco, CA: ASP), 529

Berry, D. S., Gledhill, T. M., Greaves, J. S., \& Jenness, T. 2005, in ASP Conf Ser. 343, Astronomical Polarimetry: Current Status and Future Directions, ed. A. Adamson et al. (San Francisco, CA: ASP), 71

Chandrasekhar, S., \& Fermi, E. 1953, ApJ, 118, 116

Chapin, E. L., Berry, D. S., Gibb, A. G., et al. 2013, MNRAS, 430, 2545

Coudé, S., Bastien, P., Houde, M., et al. 2019, ApJ, 877, 88

Crutcher, R. M. 2012, ARA\&A, 50, 29

Currie, M. J., Berry, D. S., Jenness, T., et al. 2014, in ASP Conf. Ser. 485, Astronomical Data Analysis Software and Systems XXIII, ed. N. Manset \& P. Forshay (San Francisco, CA: ASP), 391

Davis, L., Jr., \& Greenstein, J. L. 1951, ApJ, 114, 206

Dempsey, J. T., Friberg, P., Jenness, T., et al. 2013, MNRAS, 430, 2534

Dolginov, A. Z., \& Mitrofanov, I. G. 1976, Ap\&SS, 43, 291

Friberg, P., Bastien, P., Berry, D., et al. 2016, Proc. SPIE, 9914, 991403

Friberg, P., Berry, D., Savini, G., et al. 2018, Proc. SPIE, 10708, 107083M

Glover, S. C. O., \& Clark, P. C. 2012, MNRAS, 421, 9

Henning, T., Wolf, S., Launhardt, R., \& Waters, R. 2001, ApJ, 561, 871

Herron, C. A., Gaensler, B. M., Lewis, G. F., \& McClure-Griffiths, N. M. 2018, ApJ, 853, 9

Hildebrand, R. H. 1983, QJRAS, 24, 267

Hirashita, H., \& Li, Z.-Y. 2013, MNRAS, 434, L70

Hull, C. L. H., \& Plambeck, R. L. 2015, JAI, 4, 1550005

Jones, T. J., Bagley, M., Krejny, M., Andersson, B.-G., \& Bastien, P. 2015, AJ, 149,31

Jones, T. J., Gordon, M., Shenoy, D., et al. 2016, AJ, 151, 156

Juvela, M., Guillet, V., Liu, T., et al. 2018, A\&A, 620, A26

Kandori, R., Nagata, T., Tazaki, R., et al. 2018, ApJ, 868, 94

Kirk, J. M., Ward-Thompson, D., Palmeirim, P., et al. 2013, MNRAS, 432, 1424

Koch, P. M., Tang, Y.-W., Ho, P. T. P., et al. 2018, ApJ, 855, 39

Könyves, V., André, P., Men'shchikov, A., et al. 2015, A\&A, 584, A91

Kwon, J., Doi, Y., Tamura, M., et al. 2018, ApJ, 859, 4

Lai, S.-P., Crutcher, R. M., Girart, J. M., \& Rao, R. 2002, ApJ, 566, 925

Lazarian, A., \& Hoang, T. 2007, MNRAS, 378, 910

Lindsey, W. 1964, ITIT, 10, 339

Liseau, R., White, G. J., Larsson, B., et al. 1999, A\&A, 344, 342 
Liu, J., Qiu, K., Berry, D., et al. 2019, ApJ, 877, 43

Matthews, B. C., McPhee, C. A., Fissel, L. M., \& Curran, R. L. 2009, ApJS, 182,143

Matthews, B. C., \& Wilson, C. D. 2000, ApJ, 531, 868

Montier, L., Plaszczynski, S., Levrier, F., et al. 2015a, A\&A, 574, A135

Montier, L., Plaszczynski, S., Levrier, F., et al. 2015b, A\&A, 574, A136

Mookerjea, B., Sandell, G., Vacca, W., Chambers, E., \& Güsten, R. 2018, A\&A, 616, A31

Motte, F., André, P., \& Neri, R. 1998, A\&A, 336, 150

Müller, P., Beck, R., \& Krause, M. 2017, A\&A, 600, A63

Naghizadeh-Khouei, J., \& Clarke, D. 1993, A\&A, 274, 968

Onaka, T. 2000, ApJ, 533, 298

Ortiz-León, G. N., Loinard, L., Dzib, S. A., et al. 2018, ApJL, 869, L33

Pattle, K., Ward-Thompson, D., Kirk, J. M., et al. 2015, MNRAS, 450, 1094

Planck Collaboration, Ade, P. A. R., Aghanim, N., et al. 2015, A\&A, 576, A104

Quinn, J. L. 2012, A\&A, 538, A65

Rice, S. O. 1945, BSTJ, 24, 46

Santos, F. P., Ade, P. A. R., Angilè, F. E., et al. 2017, ApJ, 837, 161

Seifried, D., Walch, S., Reissl, S., \& Ibáñez-Mejía, J. C. 2019, MNRAS, 482,2697
Serkowski, K. 1958, AcA, 8, 135

Serkowski, K. 1962, Adv. Astron. Ap., 1, 289

Sijbers, J., den Dekker, A. J., Scheunders, P., \& Van Dyck, D. 1998, IEEE Transactions on Medical Imaging, 17, 357

Silaj, J., Jones, C. E., Tycner, C., Sigut, T. A. A., \& Smith, A. D. 2010, ApJS, 187,228

Simmons, J. F. L., \& Stewart, B. G. 1985, A\&A, 142, 100

Soam, A., Pattle, K., Ward-Thompson, D., et al. 2018, ApJ, 861, 65

Stamatellos, D., Whitworth, A. P., \& Ward-Thompson, D. 2007, MNRAS, 379,1390

Vaillancourt, J. E. 2006, PASP, 118, 1340

Vidal, M., Leahy, J. P., \& Dickinson, C. 2016, MNRAS, 461, 698

Wang, J.-W., Lai, S.-P., Eswaraiah, C., et al. 2019, ApJ, 876, 42

Wardle, J. F. C., \& Kronberg, P. P. 1974, ApJ, 194, 249

Ward-Thompson, D., Pattle, K., Bastien, P., et al. 2017, ApJ, 842, 66

Ward-Thompson, D., Pattle, K., Kirk, J. M., et al. 2016, MNRAS, 463, 1008

Weingartner, J. C., \& Draine, B. T. 2003, ApJ, 589, 289

Whittet, D. C. B., Hough, J. H., Lazarian, A., \& Hoang, T. 2008, ApJ, 674, 304

Wilking, B. A., Gagné, M., \& Allen, L. E. 2008, in Handbook of Star-forming Regions, Vol. 2, ed. B. Reipurth (San Francisco, CA: ASP), 351 